\title{
Revisiting the Route Choice Problem: A Modeling Framework Based on Mental Representations
}

\author{
Evanthia Kazagli * Michel Bierlaire * \\ Gunnar Flötteröd ${ }^{\dagger}$
}

March 29, 2016

Transport and Mobility Laboratory

School of Architecture, Civil and Environmental Engineering

Ecole Polytechnique Fédérale de Lausanne

transp-or.epfl.ch

*École Polytechnique Fédérale de Lausanne (EPFL), School of Architecture, Civil and Environmental Engineering (ENAC), Transport and Mobility Laboratory, Switzerland, \{evanthia.kazagli,michel.bierlaire\}@epfl.ch

${ }^{\dagger} \mathrm{KTH}$ Royal Institute of Technology, Department of Transport Science, 11428 Stockholm, Sweden 


\section{Abstract}

We present a new approach for modeling and analyzing route choice behavior. It is motivated by the need to reduce the complexity of the state-of-theart choice models. It is inspired by the simplifications done by the travelers, using representations of their surrounding space. The proposed framework is based on elements designed to mimic the mental representations used by travelers, denoted as Mental Representation Items (MRIs). It allows the modeler to adjust the level of complexity according to the needs of the specific application. This paper describes how operational models based on MRIs can be derived and discusses the applications of these models to traffic assignment and route guidance systems. We report estimation results using revealed preference data to demonstrate the applicability and validity of the approach.

\section{Introduction}

We are interested in modeling and forecasting the route choice behavior of individuals. Route choice ( $\mathrm{RC}$ ) is one of the key questions in travel demand analysis and at the core of traffic assignment. Route choice models (RCMs) aim at predicting the route that a given traveler would take to go from the origin of her trip to the destination. A comprehensive review of the route choice modeling problem can be found in Bovy and Stern (1990) and Frejinger (2008).

Discrete choice models (DCMs) provide a powerful and flexible methodological framework, where a great deal of explanatory variables can be considered and the heterogeneity of behavior across the population can be explicitly captured. The estimation of DCMs for route choice analysis with revealed preference (RP) data involves specific challenges. The demanding requirements in data collection and processing, the combinatorial nature of the choice set, and the structural correlation due to the physical overlap of paths (Ben-Akiva and Bierlaire, 2003) are the main issues of the estimation with RP data. These issues apply to a much lesser extent to the estimation of models with stated preference (SP) data, which is therefore easier to handle.

The conventional representation of routes is based on paths that are constructed as sequences of oriented arcs on a connected graph. In addi- 
tion to the above mentioned challenges for the modeler, the complexity of the path approach is not consistent with the actual behavior of travelers (Golledge, 1999; Taylor and Tversky, 1992). The general trend in the literature is to propose more and more complex models to deal with these challenges (Fosgerau, Frejinger and Karlstrom, 2013; Yang and Juang, 2014; Lai and Bierlaire, 2015; Ramos, 2015).

In this work, we are investigating in the opposite direction, i.e. we attempt to simplify the problem. This is accomplished by modeling the strategic decisions of people instead of the operational ones. The objective is to derive a modeling framework that (i) has a level of complexity consistent with the one handled by the travelers and (ii) attenuates the curse of dimensionality of the path-based formulation. To achieve this goal, we use modeling elements that capture the mental representations used by people throughout their travel in the transportation system. We call them Mental Representation Items (MRIs). The formal definition of the MRI elements is given in Section 3.1 .

The paper is organized as follows. Section 2 is a literature review about the challenges in route choice modeling. Section 3 is devoted to the methodology. In Section 4 we present empirical evidences of the MRI assumption and we elaborate on modeling considerations regarding data issues and applications of the model. In Section 5 we apply the methodological framework to a real case study and we illustrate its use for traffic assignment. The last section summarizes the findings of the present study and identifies the future steps of the research. 


\section{Literature Review}

Several models have been proposed to analyze route choice. The main challenge encountered by the analysts is related to the definition of the choice set. The choice set contains the alternatives that are considered by the traveler.

Three approaches are proposed in the literature. The first one relies on heuristics to construct the choice set actually considered by the travelers (Bovy and Fiorenzo Catalano, 2007; Prato and Bekhor, 2007). Examples such as the labelling approach (Ben-Akiva et al., 1984), the link elimination approach (Azevedo et al., 1993), the link penalty approach (de la Barra et al., 1993), and the constrained k-shortest path (van der Zijpp and Fiorenzo Catalano, 2005) assume deterministic shortest paths to generate the choice set. Constrained enumeration approaches based on branch-andbound are proposed by Friedrich et al. (2001), Hoogendoorn-Lanser (2005), and Prato and Bekhor (2006). However, Bekhor et al. (2006) showed that all methods fail to generate a set that is guaranteed to include the $o b$ served routes. The second approach assumes that the choice set contains all feasible paths between the origin and the destination. To make this approach operational, sampling techniques have been proposed (Frejinger et al., 2009; Flötteröd and Bierlaire, 2013). The third approach follows a technique that does not rely on sampling, while avoiding the full enumeration of paths. It has been proposed by Dial (1971) and more recently by Fosgerau, Frejinger and Karlstrom (2013), who presented the recursive logit (RL), a link-choice-based model that follows a dynamic setting which does not require choice set generation and, therefore, no sampling of alternatives either.

The second challenge is related to the modeling of the physical overlap of paths. In the context of random utility models (RUMs), it is not possible to assume that the random utilities are independent across alternatives. The approaches proposed in the literature can be divided in two categories; those dealing with the correlation in the deterministic part of the utility function, and those dealing with it in the stochastic part. Examples of the former include the C-logit proposed by Cascetta et al. (1996) and the Path Size Logit (PSL) proposed by Ben-Akiva and Bierlaire (1999). Examples of the latter include the Multivariate Extreme Value (MEV) models, such as the Paired Combinatorial Logit and Cross Nested Logit (CNL) (Vovsha 
and Bekhor, 1998; Lai and Bierlaire, 2015), and Non-MEV models, such as the Probit (Daganzo and Sheffi, 1977) and the Logit Kernel model (Bekhor et al., 2002; Frejinger and Bierlaire, 2007).

Dealing with correlation in the stochastic part of the utility increases the model complexity and entails difficulties with respect to the estimation, especially for large networks. Frejinger and Bierlaire (2007) introduced the concept of subnetworks within a factor analytic specification of an error component model. The authors argue that they capture correlation in a behaviorally realistic way without increasing the complexity of the model. Yet, the estimation of such a model for large networks is cumbersome.

Mai et al. (2015) exploit the RL model by Fosgerau, Frejinger and Karlstrom (2013) and extend it to the nested RL. The latter builds on the RL model, where no choice set generation is needed, and improves it to accommodate the correlation of path utilities. The first work to use a MEV model with sampling of alternatives is the one by Lai and Bierlaire (2015). The authors specify a CNL model and adopt the Metropolis-Hastings algorithm proposed by Flötteröd and Bierlaire (2013) with a new expansion factor inspired by Guevara and Ben-Akiva (2013) in order to avoid the enumeration of paths.

Most models cited above are path based. The concept of path is evidently hard to handle due to the operational limitations discussed above -path generation, sampling, complex correlation- but also due to the fact that drivers do not actually make travel plans decisions based on paths (Golledge, 1999). The latter entails behavioral limitations. Hence, the state-of-the-art models are either very complex or often fail to capture observed behavior. No realistic yet simple model based on RP data has been proposed. This is where the contribution of the present study lies. We propose a simple model, which is not based on paths, and exploits RP data. It uses elements emulating the mental representations that are formed by travelers.

Intuitively speaking, if we ask a traveler to describe her itinerary from home to work, we do not expect her to report sequences of links. A few attempts to use perceptual concepts in route choice analysis include the labelling approach by Ben-Akiva et al. (1984) for path generation and sampling, and the subnetworks approach by Frejinger and Bierlaire (2007) to capture correlation. Yet, the scope of these works is different and the modeling element is still a path. In this work we try to identify the strategic 
decisions of the travelers that are associated with their mental representations of the area of interest.

The literature of cognitive science, environmental psychology and geography, assisted in gaining insights into the representation of large-scale environments and spatial behavior. It includes, but is not limited to, Tolman (1948), Lynch (1960), Suttles (1972), Chase (1983), Couclelis et al. (1987), Golledge (1999), Golledge and Gärling (2003), Arentze and Timmermans (2005), Hannes et al. (2008).

In these research fields, and in their attempts to answer questions such as how people perceive and process information during travel in spatial networks, we come across concepts such as the mental map, the mental representation, or the anchor point. Each field approaches these concepts from a different perspective and defines them accordingly. Lynch (1960) decomposes the image of the city into paths, edges, districts, nodes and landmarks. Suttles (1972) defines a cognitive map as the mixture of qualitative and spatial information that allows us to make decisions in a spatial context. Golledge (1999) argues that individuals relate to anchor points in the spatial environment and that the anchors have a dual role: (i) they serve as organizing elements of peoples' mental maps, and (ii) they enable way-finding. Recent work by Hannes et al. (2008), defines the mental map as "The whole of spatial and travel related information used and stored in memory".

Contrary to the research conducted in the disciplines cited above, this study does not look at how the representations of space are formed or learned. It rather exploits the intuition gained from these fields in the effort to build a flexible and operational framework for route choice analysis. 


\section{Methodological Framework}

In this section, we outline the methodology for the definition of a route choice model based on mental representations. In addition to the mental representations themselves (Section 3.1), four main elements need to be defined for the development of an operational random utility model:

1. the choice $\operatorname{set} \mathcal{C}_{n}$ (Section 3.2),

2. the specification of the utility functions (Section 3.3),

3. the likelihood function and the measurement equations that are necessary for the estimation of the model parameters (Section 3.4), and finally

4. the mapping of the modeling elements to the elements of the application of the route choice model (Section 3.5).

The key feature of the present framework is the representation of routes as sequences of Mental Representation Items (MRIs). Contrary to the current state-of-the-art RCMs, the MRI framework is of greater generality. For instance, it is possible to define a MRI model that is independent from a network model.

In what follows, we start by providing a formal definition of the MRI and proceed with a description of the procedure for the specification, estimation and application of an operational MRI model.

\subsection{The Mental Representation Item}

A "Mental Representation Item" is a modeling element that captures any perceived items influencing a route choice. It is formally characterized by:

1. a name,

2. a description,

3. a geographical span, and

4. a list of representative geocoded points.

The first two components capture the conceptual aspects, and the last two are needed for the model to be operational. 


\subsubsection{The MRI components}

Fig. 1 provides an illustration of the four MRI components in the context of a route choice problem in the city of Athens, where the choice consists in going through the city center or avoiding it.

In what follows, we briefly discuss the purpose and use of each component and refer the reader to Section 4 for further insights and examples.

\section{Conceptual components}

1. The name is the label of the MRI that is determined based on a toponym, such as "Katechaki" in Fig. 1. It is similar to the identification of a link in a network model. The difference is that the name of the MRI is not merely based on a numbering convention -as it is the case with the link identification. It actually characterizes the modeling element.

2. The description of the MRI completes the conceptual definition of the modeling element. It is typically a wording that would be used by a traveler describing the itinerary to somebody, such as the "Peripheral" in Fig. 1.

Operational components The operational components are designed to associate the MRI with a more objective representation, such as a map or a network.

3. The geographical span of the MRI may be an area, a polyline, or any other shape, convex or not, that determines the boundaries of the MRI on a map or a network model. If a network model is available the span may also be defined as a list of links and nodes. If not, it can be defined as a geomarked element on a map. The geographical span allows the analyst to relate the observations to MRIs.

4. A list of representative points. A representative point is any point or item that is characteristic for the MRI. It may be any landmark in the area of interest. Depending on the scope of the analysis, a representative point may be a major intersection in a city, or a city itself -for urban and interurban route choice analysis, for example. 


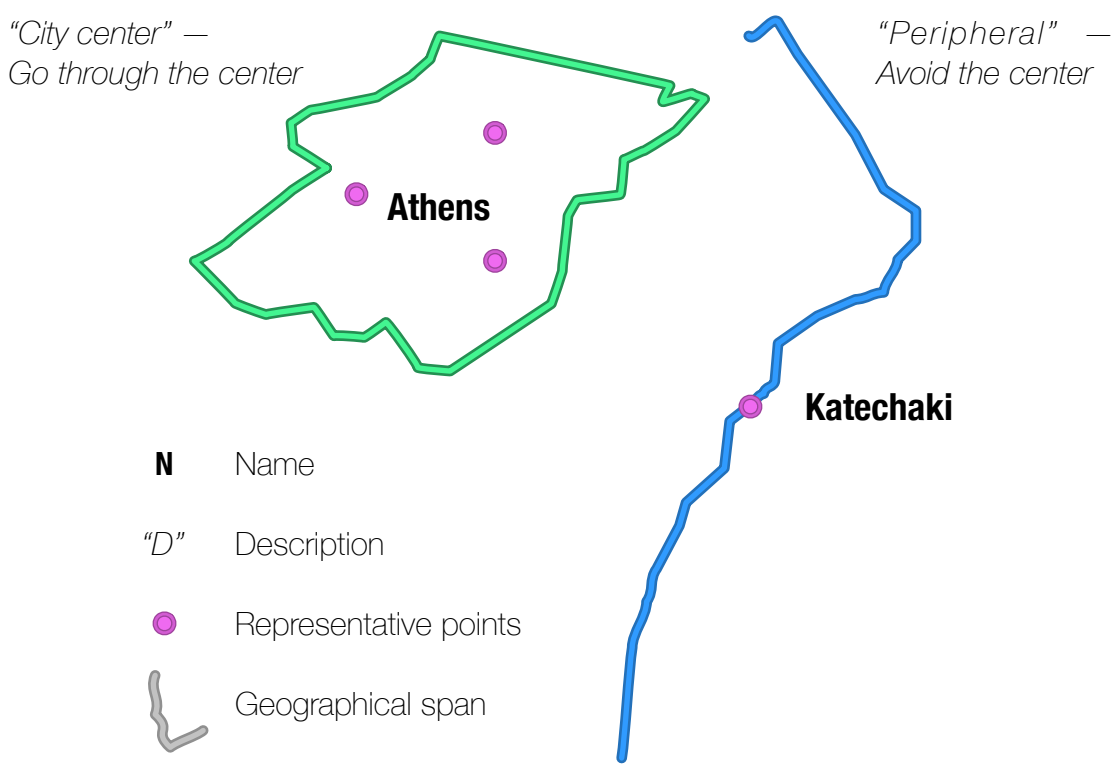

Figure 1: Examples of two MRIs and their components.

The representative points play a central role in the model specification as described in Section 3.3.

\subsubsection{Modeling considerations}

A MRI is an item characterizing the mental representation of an itinerary. Defining the MRI is a similar exercise as defining the alternatives of a choice model. It is context dependent and it may be complex. In the context of route choice, the complexity may depend on the size of the city, the dispersion of the OD pairs, etc. The key difference is that the level of complexity is not constrained by the network topology as in a pathbased formulation. The analyst has the flexibility to select the right level of complexity, consistently with the behavioral assumptions and the type of application of the model.

The definition of the MRI is the output of modeling considerations that draw insights from the relevant literature, the expert's kwonledge of the area of interest and, ideally, travelers' description of their itineraries.

The MRI must be designed such that (i) it has a meaningful behavioral interpretation reflected by the conceptual components and (ii) its level 
of aggregation is high enough for the model to be simple and operational yet low enough for the model to be useful (this is reflected by the the operational components). As a rule of thumb, the analyst should keep in mind that defining the MRIs to be geographically disjoint would allow for simpler model structures. It is also advisable to keep the number of MRIs low so that the number of MRI sequences is also low and can be enumerated to characterize the choice set.

\subsection{Definition of the alternatives}

Following the definition of the MRI, a route is defined as:

1. an origin,

2. an ordered sequence of MRIs (possibly only one), and

3. a destination.

An advantage of the alternatives generated on the basis of MRI sequences is that they potentially have a much simpler correlation structure than the path alternatives. The case study presented in Section 5 deals with the simplest possible case, where each route is described by a single MRI and there is a common choice set for all individuals.

\subsection{Specification of the utility functions}

As soon as the MRIs are defined and the choice set $\left(\mathcal{C}_{n}\right)$ is determined, we are able to specify a route choice model and define the choice probabilities $\mathrm{P}\left(\boldsymbol{i} \mid \mathcal{C}_{n}\right)$ for each alternative $i$. Like for any random utility model, each alternative is associated with a utility, which is a function of the attributes of the alternatives $x_{i n}$ and the characteristics of the individual $z_{n}$. Once the utility functions have been defined, any choice model $\mathrm{P}\left(\mathrm{i} \mid \mathcal{C}_{n}, x_{i n}, z_{n}\right)$ (logit, nested logit, cross-nested logit model, etc.) can be considered.

As the main modeling elements are mental representations, the alternatives and their attributes are in general latent, that is, based more on perceptions than on objective measurements. The exact way to deal with this issue is application dependent. In Section 5, we present a model that is simple to develop and to use. We also propose below three simple heuristics to generate attributes for each alternative. The third one does not require 
a network model. Clearly, these are just simple examples, and more advanced specifications, exploiting the literature on perceptions, could be investigated as well.

A deterministic approach with representative paths We assume here that a network model of the area of interest is available, and that the representative points of each MRI are nodes of this network. A unique representative path is associated with each alternative (Fig. 2), where two consecutive points are connected by the shortest (or the fastest) path. The procedure consists of the following steps prior to the estimation of the model:

1. For each MRI and each choice context, select one of the representative points corresponding to a node in the network. The selected representative point can be different for different OD pairs, or for different individuals. Subsequently, for each individual and each alternative, there is a sequence of nodes connecting the origin and the destination of the trip.

2. Generate one path connecting the nodes. Typically, this can be done by connecting each pair of successive nodes by the shortest path between them according to a given metric, such as length, travel time, or any relevant performance measure. This path is the representative path of the alternative.

3. The utility function of an alternative is defined as the utility function of the representative path that would be used in a classical route choice model.

This is the approach that we have applied for the case study presented in Section 5. Our point is to show that such a simple approach can provide meaningful results, although we acknowledge that more sophisticated procedures may be more appropriate. For instance, the stochastic extension of this procedure described below.

A stochastic multi-path approach A direct generalization of the heuristic presented above would be to generate a list of paths associated with each sequence of MRIs. Each of these paths would be associated with a utility 


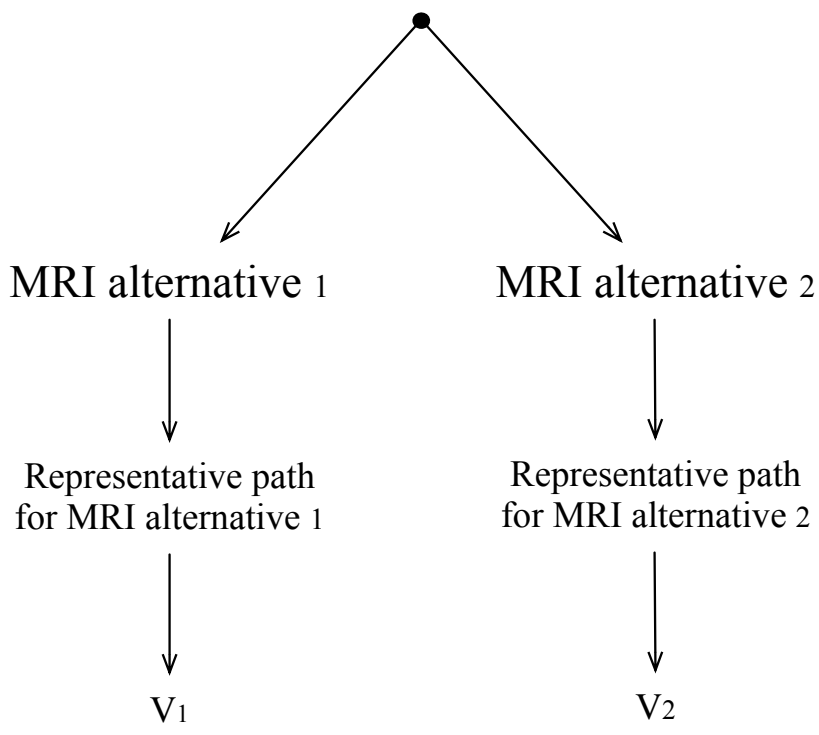

Figure 2: Schematic representation of the specification of utility functions through representative paths.

function, in a way similar to a classical path-based route choice model. Based on the utility maximization principle, the utility associated with the alternative would be the highest utility among these paths. The deterministic part of this utility is then given by the expected maximum utility. If a MEV model is considered, generated by the function $G$, the expected maximum utility is given by $\ln (\mathrm{G})$ (see Fosgerau, McFadden and Bierlaire (2013)).

Network-free approach In the MRI framework, it is not necessary to use a network model and paths. As each MRI is associated with a geographical span, it is possible to use attributes that are associated with a geographical area, such as the density of landmarks or points of interest. For example, Boarnet et al. (1998) propose a congestion index associated with geographical areas. Along the same lines, and in the context of pedestrian route choice, Frank et al. (2009) propose a walkability index. The use of the MRI framework in a network free context opens new research opportunities for route choice analysis and applications. 


\subsection{Model estimation}

The estimation of the parameters of the model is accomplished by maximum likelihood estimation. In the MRI framework, the available data may not be expressed in terms of the MRIs. Consequently, the development of measurement equations is necessary.

\subsubsection{Measurement equations}

To estimate a MRI choice model, a measurement equation is needed that captures the contribution of each piece of data to the likelihood function. Let $i$ be an alternative of the MRI model (that is an ordered sequence of MRIs) and $y$ be an observation -it can be a reported sequence of places in a survey, or a GPS trace. Its contribution to the likelihood function is then

$$
\sum_{i \in \mathcal{C}_{n}} \mathrm{P}(y \mid \mathfrak{i}) \mathrm{P}\left(\mathfrak{i} \mid \mathcal{C}_{n}, x_{i n}, z_{n}\right)
$$

where $\mathrm{P}\left(\mathfrak{i} \mid \mathcal{C}_{n}, x_{i n}, z_{n}\right)$ is the MRI choice model, and $\mathrm{P}(\mathrm{y} \mid \mathfrak{i})$ is the measurement equation. The latter models the data generation process, and is the probability to observe $y$ given that the alternative $i$ is actually chosen by the traveler. The exact definition of such measurement equations is application dependent. An example is provided in Section 5. In general, the MRI' s geographical span (Section 3.1.1) plays an important role in the specification of the measurement equations. In some circumstances, it is possible to associate each piece of data with a single alternative, so that $\mathrm{P}(\mathrm{y} \mid \mathrm{i})$ has values 1 , if the observation $y$ traverses the geographical span of the MRI (Fig. 3), and 0 otherwise. In that case, equation (1) involves only one term. Examples of measurement equations can be found in Bierlaire and Frejinger (2008) and Chen and Bierlaire (2013).

\subsection{Model application}

The generality of the MRI framework may not allow a direct application of the model in a desired context. Similar to the necessity of measurement equations for the model estimation, it may be necessary to relate the latent constructs associated with the MRIs to concrete elements for a given application. To illustrate that, consider the traffic assignment problem $-\mathrm{a}$ 


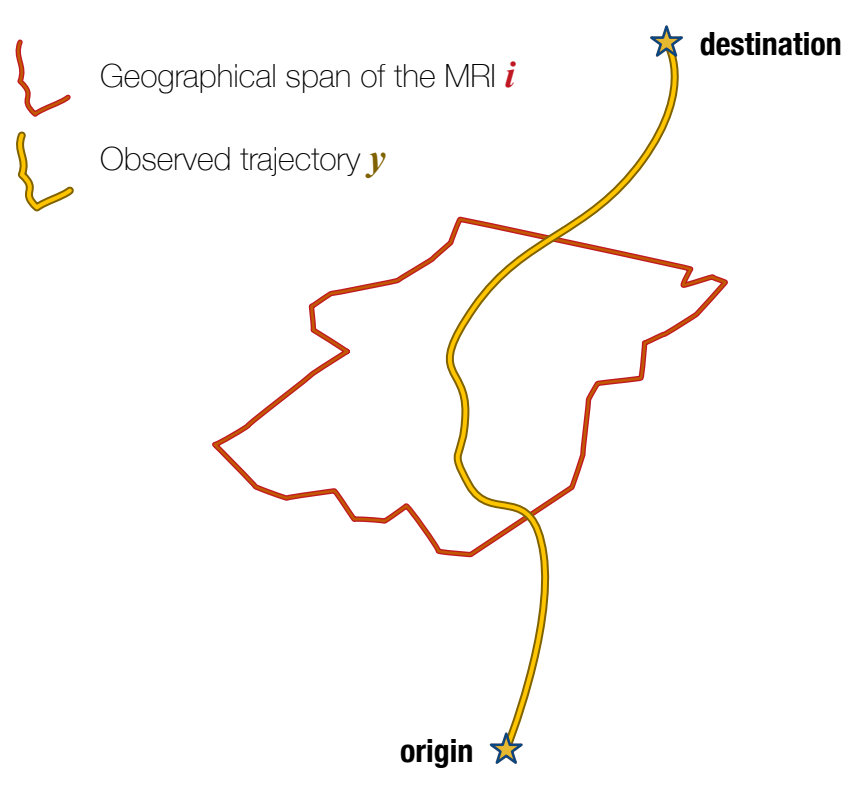

Figure 3: Illustration of $\mathrm{P}(y \mid i)=1$.

typical application of a route choice model. In this paragraph, we discuss operational aspects of using the MRI model in this context.

Let us consider the assignment of a single traveler $n$ with known origin and destination. We are interested in the probability $\mathrm{P}\left(\mathrm{a} \mid \mathcal{C}_{n}\right)$ that traveler $n$ crosses any network link a, given her choice set $\mathcal{C}_{n}$. In order to exploit the MRI choice model, this probability is written as

$$
\mathrm{P}\left(\mathrm{a} \mid \mathcal{C}_{\mathfrak{n}}, x_{\mathrm{in}}, z_{\mathfrak{n}}\right)=\sum_{i \in \mathcal{C}_{\mathfrak{n}}} \mathrm{P}(\mathrm{a} \mid \mathfrak{i}) \cdot \mathrm{P}\left(\mathfrak{i} \mid \mathcal{C}_{\mathfrak{n}}, x_{\mathrm{in}}, z_{\mathfrak{n}}\right)
$$

where $P\left(i \mid \mathcal{C}_{n}, x_{i n}, z_{n}\right)$ is the MRI choice model, and $P(a \mid i)$ is the probability of using link a given that alternative $i$ is chosen. $P(a \mid i)$ can be seen as the operational component, that is the implementation of decision $i$, while $\mathrm{P}\left(i \mid \mathcal{C}_{\mathfrak{n}}, x_{\mathrm{in}}, z_{\mathfrak{n}}\right)$ is the behavioral component represented by the MRI choice model. One possibility to specify $\mathrm{P}(\mathrm{a} \mid \mathfrak{i})$ consists in using a path-based representation. In that case, the specification would be

$$
P(a \mid i)=\sum_{p} 1(a \in p) \cdot P(p \mid i)
$$


where $\mathbf{1}(a \in p)$ is the zero/ one indicator of path $p$ containing link $a$ and $P(p \mid i)$ is the probability of traveling along path $p$ given that MRI $i$ is chosen. For the sake of illustration, a simple model specification is described in Appendix B. It is used in Section 5.6 to illustrate the application of the MRI model to traffic assignment for the case study of interest. 


\section{Additional Considerations}

In this section, we provide additional considerations that may be useful when developing and applying the MRI approach.

\subsection{Defining the MRI}

The flexibility of the framework allows to use various sorts of data for the estimation of the model. But data is also useful to drive the model specification and, in particular, the definition of the MRIs. Conducting interviews and surveys is particularly useful in this context. Reported itineraries are an ideal data source. In this paragraph, we present an example of a small qualitative survey providing insights for the MRI assumption.

In order to investigate how mental representations could be exploited for modeling purposes, we interviewed three drivers in the cities of Athens and Stockholm. Respondents were asked to give a description of the routes that they follow to go from home to work, or to a relative's place. We are interested in the wording they use to describe the itinerary.

All the respondents have good knowledge of the network and, if asked explicitly, they are able to describe the exact itinerary they follow in details, for instance: "I go right in the first traffic light, continue straight for about 300 meters and turn left in the third traffic light that I encounter.". However, in their initial response to the question Describe your itinerary from home to work, they never give detailed itineraries. Instead, they identify two to three alternatives that they choose in rotation depending on the time of day -indicating different expectation of congestion- that are always associated with some conceivable element of the city, such as the city center, the highway $H$, the neighborhood $N$, the bridge $B$. These elements are used to identify alternatives. In some cases they also identify an alternative that they never choose; e.g. entering specific areas that are in the congestion pricing zone in Stockholm.

An example of a described itinerary is: "I take $E_{4}$ (major highway traversing Sweden) and then enter the city from the entrance in Solna (one of the main municipalities in Stockholm). I avoid Södermalm (district in central Stockholm) because of the tolls.", or "I go through Arsta (district in Stockholm) and then take the bridge to Kungsholmen (one of the islands that Stockholm comprises of).". The comparisons 
of these alternatives are described as "longer but faster", "faster because of less traffic lights", "more pleasant", "more boring", etc., meaning that not only the routes but also their attributes are perceived in an aggregated manner.

For the case of Athens, it appears that a natural example of a MRI is "the city center". From the example of Stockholm, a possible MRI would be a bridge. Indeed, Stockholm spreads across 14 islands, which are connected through bridges and tunnels.

Figure 4 illustrates a few examples of MRI sequences in the city of Stockholm. Three alternatives, as identified by one of the interviewed drivers (see Section 4.1), are depicted. Two of the alternatives first cross the district of Arsta and then entail a bridge choice either through Essingeleden, or through Liljeholmensbron, while the third alternative passes by the Gullmarsplan square (metro station), traverses the Söderledstunneln and then goes through the city center.

The experiments conducted in the two cities support our hypothesis that choice takes place at a higher conceptual level and that the exact sequence of links, related to the concept of path, is just the implementation of this choice. In Appendix A we present one of the interviews for the city of Athens in details. Through the interviews we get (i) insight into peoples' perception of route options, and (ii) intuition of how to define MRIs in a behaviorally realistic way.

\subsection{Model-to-data approach}

A MRI aims at representing how travel options are grouped and perceived; it does not necessarily aim at representing groups of realized travel patterns. Clustering GPS track segments may be a possible approach to defining MRIs if both groupings -of perception and realization- overlap. However, this is in general difficult to ascertain. Hence, the concrete choice of a MRI structure should be left to the modeler.

In the context of GPS records, we do not suggest a data-driven approach that blindly extracts MRIs from the records. Instead of answering the question "How to define the MRIs given the availability of GPS data?", we suggest to address the question "Is the definition of the MRIs consistent

with the observations?" Our approach can be seen as "confirmatory", as opposed to "exploratory". 


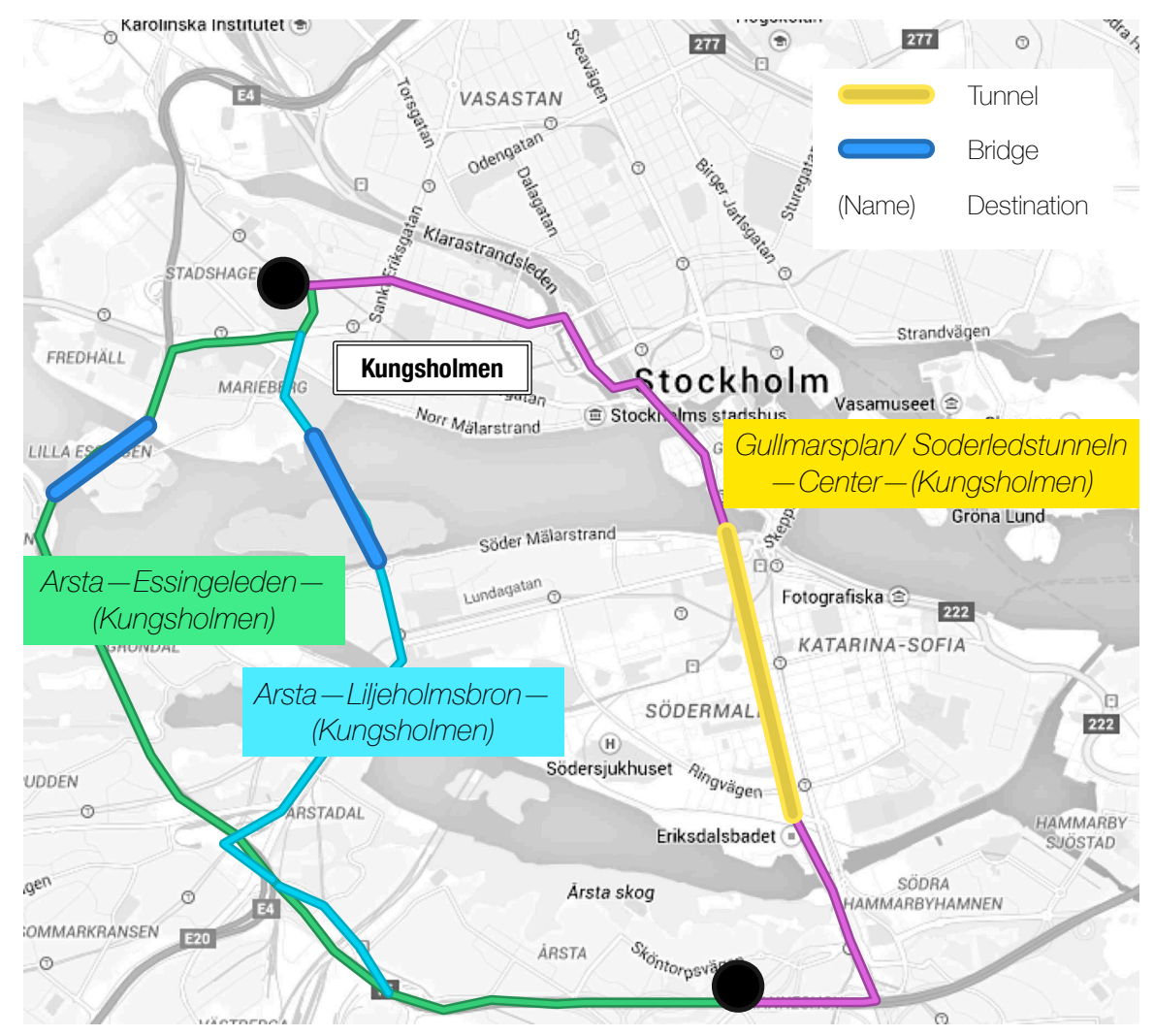

Figure 4: Examples of MRI sequences in the case of Stockholm.

\subsection{Route guidance}

The proposed MRI approach is particularly relevant in the context of route guidance systems. The nature of the MRI makes it a natural way to provide information and guidance directly to users, without the necessity to use a navigation system. Variable Message Signs (VMS), radio announcements, oral instructions can easily benefit from the output of the model. Still, the models can be used for navigation systems using the same decomposition as described in Section 3.5.

At the behavioral level, a recommendation of the sequence of MRIs is provided "to go to the airport, avoid the city center and use the bridge". If this recommendation must be transformed into a path, methods similar to those described above, exploiting the representative geocoded points, can be considered. Note that current navigation systems already work with this concept: if one provides as a destination the name of a city, without being 
more specific, the system itself selects a specific location in the city center and leads one there.

With the help of the MRI-based behavioral model, in-vehicle navigation systems could be adjusted according to the needs of the driver. As an example, drivers with good knowledge of the network do not always need step-by-step instructions to reach their destination, rather suggestions that would help them to avoid congestion in specific parts of the network (e.g. avoid the city center). In this context, the navigation systems could provide the option to choose between detailed itineraries in case of new destinations, or aggregate route suggestions in case of everyday trips, such as the trip to work, according to the current traffic conditions. We provide such an example in Section 5.6, following the traffic assignment illustration. 


\section{Case Study}

The objective of this case study is to demonstrate that the model is indeed applicable with real data. We focus here on a case where only GPS data is available, and we show that, despite the need to use heuristics that may sound arbitrary in the first place, sensible results can be obtained. For this purpose, we use the network of Borlänge in Sweden (Fig. 5). The network consists of 3077 nodes and 7459 unidirectional links. The estimation results presented in this section are based on real GPS data collected from private vehicles in the city of Borlänge. The data had been previously processed to obtain map-matched trajectories useful for route choice analysis ${ }^{1}$. Each observation consists in a sequence of links from the origin to the destination node.

In what follows, we present one possible way to operationalize the model, according to Section 3, taking advantage of the available network model. Note that, consistently with the objective of this research, we have tried on purpose to keep the modeling as simple as possible.

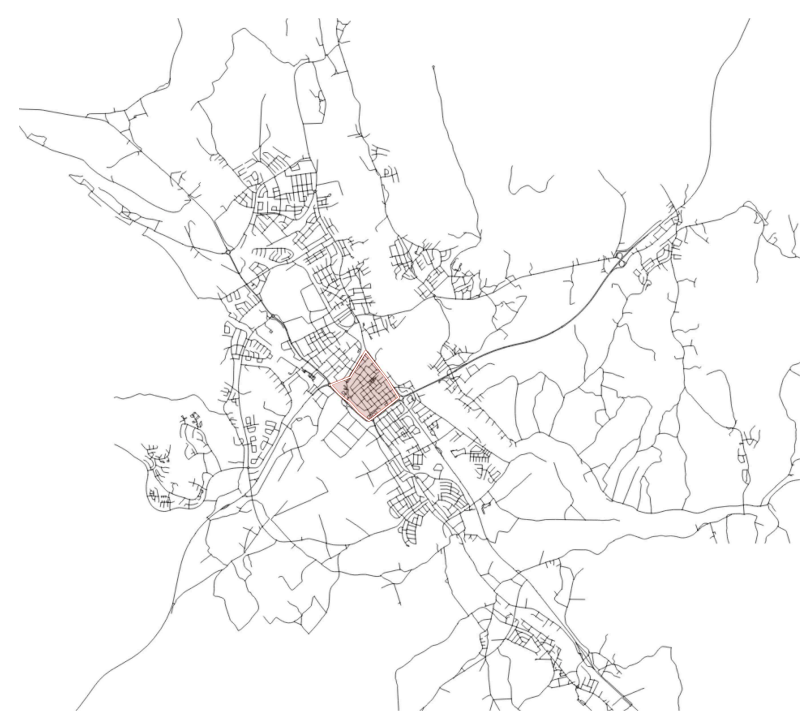

Figure 5: Borlänge road network where the city center is highlighted by the shaded area.

\footnotetext{
${ }^{1}$ We refer to Frejinger and Bierlaire, 2007 and Axhausen et al., 2003 for a description of the Borlänge GPS dataset.
} 


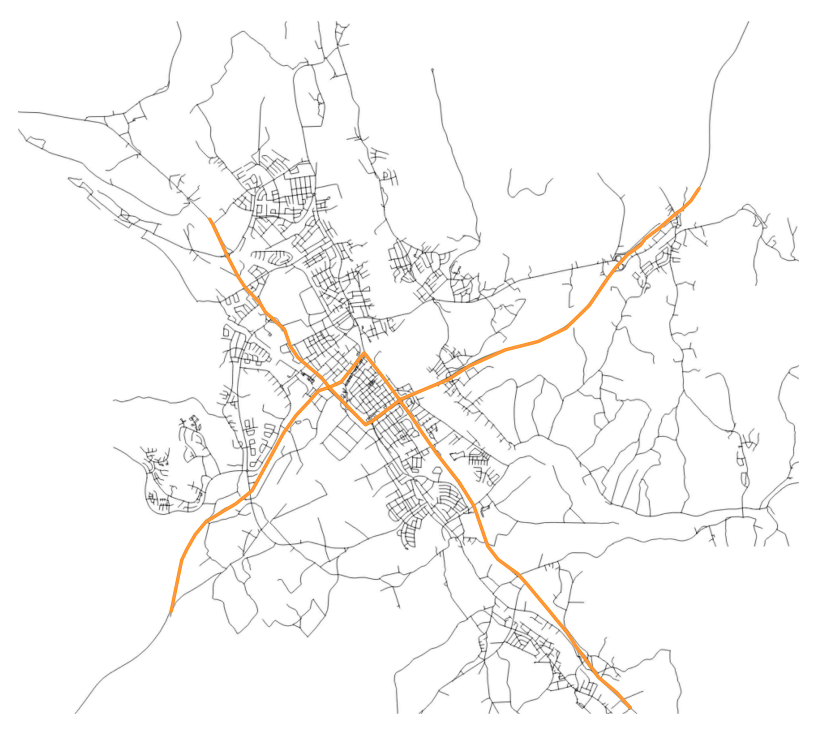

Figure 6: National roads, R50 and R.70, in Borlänge encircling the city center.

\subsection{Definition the choice problem}

In lack of survey data where people talk about their itineraries, we merely rely on examining the network of Borlänge for the definition of the MRIs.

The center of the city (shaded area in Fig. 5) is the first distinct element in the network of Borlänge. It is characterized by higher density of small streets in comparison with the rest of the network. This core is encircled by the national roads (R.50 and R.70) that are highlighted in Fig. 6. In most of their parts, the national roads have four lanes -two in each directionand the pavement is divided by guard-rails. These features signify higher operating speeds, as well as higher convenience, in comparison with the rest of the streets that have at most one lane per direction, lower speed limits, and are less direct.

Figures 7 and 8 show the output of Google Maps Directions API for route options given two arbitrarily selected OD pairs. After investigating options proposed by Google Maps for several OD pairs, it seems that three high level possibilities appear: (i) going through the city center, (ii) following orbital routes around the city center and along its boundaries, and (iii) avoiding the city center. Examples of each option are provided in Fig. 


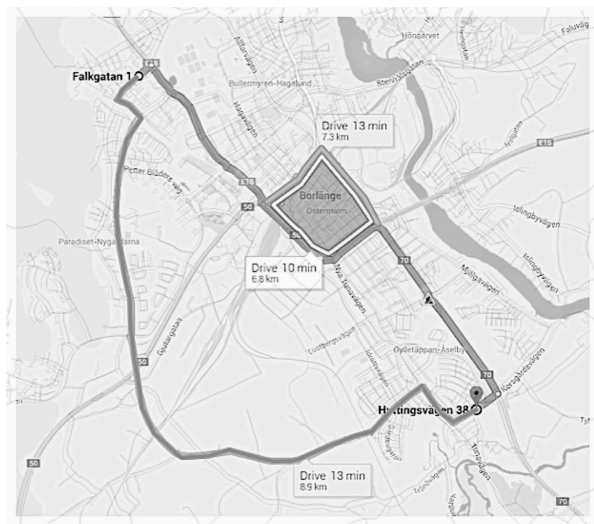

Figure 7: Example of route options provided by Google Maps Directions.

7 and 8. These patterns have also been observed in the dataset.

Consequently, we identify three major elements: the "city center", the "perimeter of the city center", and the "avoid the city center". We further separate the "perimeter of the city center" in two elements: (i) "clockwise movement", and (ii) "counter-clockwise movement", indicating left and right turn accordingly. These are two clear options for travelers as they approach the center. Therefore, we obtain four MRIs. We label them as CC, CL, CO and AV, equivalently for the "city center", the "clockwise movement", the "counter-clockwise movement" and the "avoid the city center".

We now characterize the four MRIs. According to Section 3.1.1:

1. the name for the

(a) CC corresponds to the "city center of Borlänge",

(b) $\mathrm{CL}$ and $\mathrm{CO}$ corresponds to the name of the streets defining the perimeter; these are the Backaviadukten (south half of the perimeter), Siljansvägen (north-east part of the perimeter), and Ovanbrogatan (north-west part of the perimeter),

(c) AV may correspond to the name of any street that can be used to avoid the center.

2. the description for the

\footnotetext{
${ }^{2}$ Note that there is the river on the east side of the city. In the present work we do not consider trips between the two sides of the river.
} 


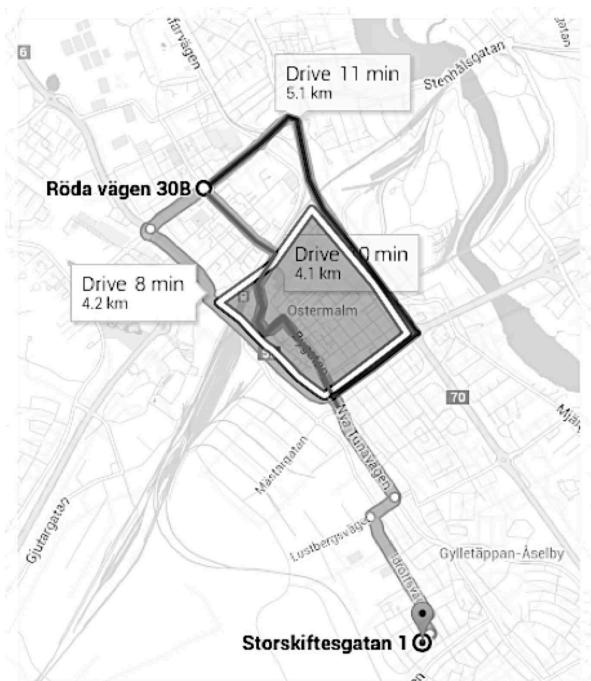

Figure 8: Example of route options provided by Google Maps Directions.

(a) CC would correspond to a sentence equivalent to "go through the center",

(b) CL would correspond to "turning left" as approaching the city center,

(c) $\mathrm{CO}$ would correspond to "turning right" as approaching the city center,

(d) AV would correspond to a sentence equivalent to "take the peripheral $X$ to avoid the city center".

3. the geographical span for the

(a) CC is the list of all links of the network that are inside the perimeter that defines the boundaries of the center,

(b) CL and CO is the list of all links on the perimeter defining the boundaries of the center,

(c) AV is the list of all the remaining links, that is, excluding the ones inside and on the perimeter of the center.

4. the list of representative geocoded points (Fig. 10) for the

(a) CC consists in the two nodes that roughly correspond to the middle points of the two main streets in the center (Fig. 9), 
(b) CL and CO consists in the three nodes that roughly correspond to the middle points of the three streets.

(c) AV consists in the two nodes that correspond to the two main peripherals that can be used to avoid the center, depending on the origin and destination of the trip.

Once the MRIs are identified, the choice set must be built. We consider the most simple case: each alternative involves exactly one MRI. Moreover, the choice set is the same for every individual in the population that we consider. Therefore, we have $\mathcal{C}_{n}=\{\mathrm{CC}, \mathrm{CL}, \mathrm{CO}, \mathrm{AV}\}$, for all $\mathrm{n}$.

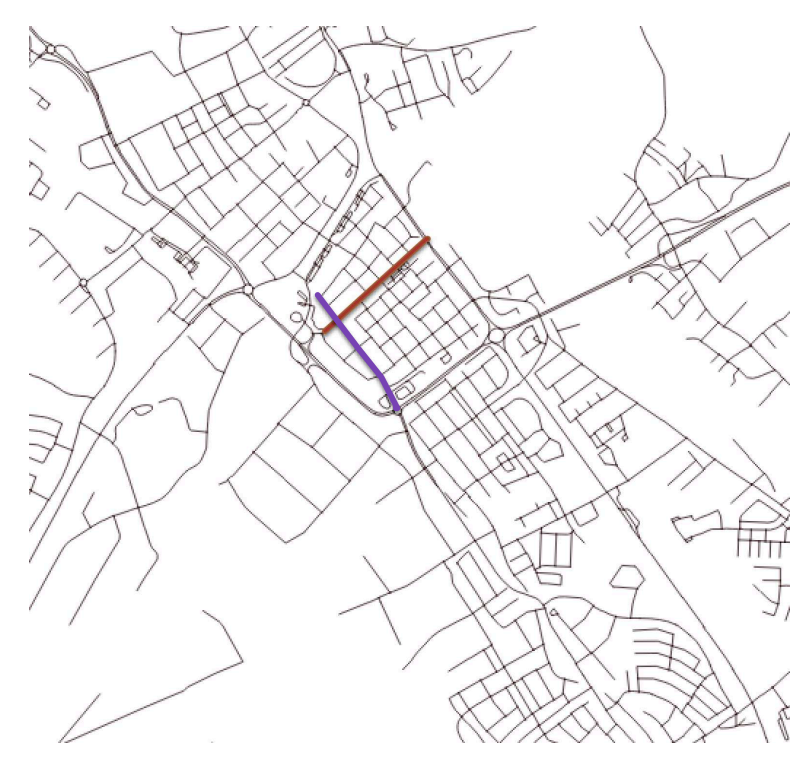

Figure 9: The two main streets in the city center. 


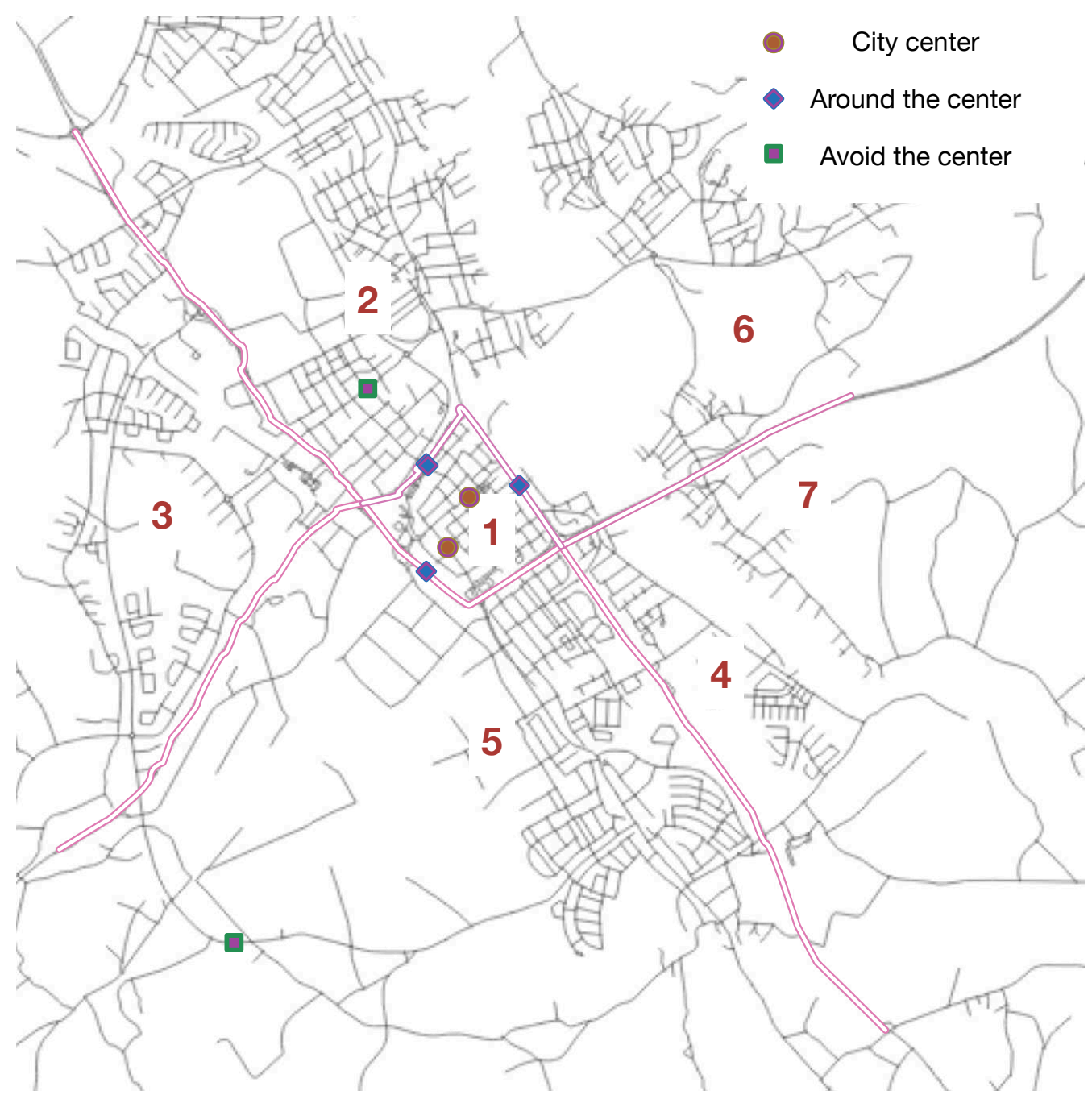

Figure 10: Representative points of the MRIs. 


\subsection{Model specification}

The fact that the MRIs consist of physically disjoint network elements justifies the use of a logit model. We compare two different specifications. The first specification includes travel times and the second one lengths. Tables 1 and 2 show the specification of Model 1 and Model 2, respectively. We include the variables number of left turns (LEFT) and number of intersections (IS) in both specifications. As the choice set size is four it is possible to estimate ASCs. These specifications have been obtained after testing various specifications.

Table 1: Specification table of Model 1 with piecewise linear travel time

\begin{tabular}{|c|c|c|c|c|}
\hline Parameter & $\mathrm{CC}$ & $\mathrm{CL}$ & $\mathrm{CO}$ & AV \\
\hline $\mathrm{ASC}_{\mathrm{CC}}$ & 0 & 0 & 0 & 0 \\
\hline $\mathrm{ASC}_{\mathrm{CL}, \mathrm{CO}}$ & 0 & 1 & 1 & 0 \\
\hline$A S C_{A V}$ & 0 & 0 & 0 & 1 \\
\hline$\beta \operatorname{TIME}_{\mathrm{CC}}$ & $\mathrm{TT}(\min )$ & 0 & 0 & 0 \\
\hline$\beta T \mathrm{TME}_{\mathrm{C}(0 \mathrm{~min})}^{(0-10 \mathrm{~m}}$ & 0 & $\mathrm{TT}(\min ) \leq 10$ & $\mathrm{TT}(\min ) \leq 10$ & 0 \\
\hline$\beta T I M E_{C L C O}^{(1 / 0 \mathrm{~min})}$ & 0 & $\mathrm{TT}(\min )>10$ & $\mathrm{TT}(\min )>10$ & 0 \\
\hline$\beta T I M E_{A V}$ & 0 & 0 & 0 & $\mathrm{TT}(\min )$ \\
\hline$\beta$ LEFT & \# left turns & \# left turns & \# left turns & \# left turns \\
\hline$\beta I S$ & \# IS & \# IS & \# IS & \# IS \\
\hline
\end{tabular}

Table 2: Specification table of Model 2 with the length formulation

\begin{tabular}{|c|c|c|c|c|}
\hline Parameter & $\mathrm{CC}$ & CL & $\mathrm{CO}$ & AV \\
\hline $\mathrm{ASC}_{\mathrm{CC}}$ & 0 & 0 & 0 & 0 \\
\hline $\mathrm{ASC}_{\mathrm{CL}, \mathrm{CO}}$ & 0 & 1 & 1 & 0 \\
\hline$A S C_{A V}$ & 0 & 0 & 0 & 1 \\
\hline$\beta$ LENGTH $\mathrm{CC}$ & Length (km) & 0 & 0 & 0 \\
\hline$\beta$ LENGTH ${ }_{\mathrm{CL}, \mathrm{CO}, \mathrm{AV}}$ & 0 & Length $(\mathrm{km})$ & Length $(\mathrm{km})$ & Length $(\mathrm{km})$ \\
\hline$\beta$ LEFT & \# left turns & \# left turns & \# left turns & \# left turns \\
\hline$\beta I S$ & \# IS & \# IS & \# IS & \# IS \\
\hline
\end{tabular}




\subsection{Data processing and measurement model}

The available dataset of GPS map-matched trajectories needs to be transformed into a dataset consistent with the model specification. In this case, the measurement model defined in (1) is deterministic, and each observation is replaced by one alternative of the MRI model, according to the definition of the geographical span (see Section 5.1). More precisely, $\mathrm{P}(\mathrm{y} \mid \mathrm{i})=1$, if the observed path traverses the geographical span of the MRI, and zero otherwise. Note that in this application, each observation traverses exactly one MRI. Examples of observed routes, and the MRI that they correspond to, are provided in Fig. C1a-e. This step was initially done manually in order to gain insight into the data and develop the rules to automate the procedure.

The network is divided in 7 zones but for this case study we only use 5 zones. We ignore trips where either the origin or the destination of the trip is located in the other side of the river (zones 6 and 7). We obtain a sample of 139 observations for the estimation of the model.

To facilitate the determination of the representative node $r$ for each observation a simple zoning system is assumed (Fig. 10). As an example, avoiding the center usually corresponds to a long detour (Fig. C1e). Indeed, for people traveling from south to north, and vice versa, this is the only way to avoid the center (through Paradisvägen, a rural street). Only for trips between the zones 2 and 3 it is possible to avoid the center through other streets. For these trips we define a second representative node ${ }^{3}$.

We use the heuristic described in Section 3.3 for the specification of the utility functions. The representative path for each MRI is the fastest path. A representative example of the four alternatives in the MRI choice set, as generated by this process, is depicted in Fig. 11.

We remark that the operationalization of the MRI utility functions is dependent on the selection of the representative points. In the case of Borlänge, the network is relatively small and the identification of the representative points was relatively straightforward. For more complex networks, a sensitivity analysis, demonstrating the effects of using different points on the results might be necessary.

For the network model of Borlänge the available information includes

\footnotetext{
${ }^{3} \mathrm{An}$ additional MRI alternative could be considered in this case, but due to the low number of observations we did not proceed with this demarcation.
} 


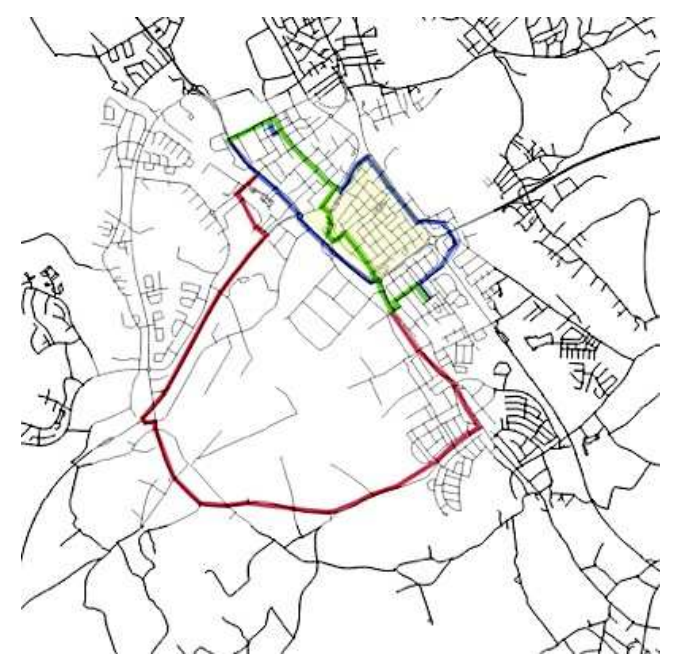

Figure 11: Illustration of a MRI choice set with the representative paths.

the link lengths, the turning angles between each pair of links and the inand out-degree of each node, allowing us to identify intersections. The travel time of each link is computed as the ratio of the link length divided by an average speed, the latter being the average observed speed over all observations and all links with the same speed limit. Statistics on the attributes of the MRI alternatives are provided in Table 3. Table 4 shows the number of times that each MRI is chosen. Figure 12 shows the distribution of the travel times of the four MRI alternatives.

\subsection{Model estimation}

The parameter estimates ${ }^{4}$ are presented in Table 5. All the parameters have the expected signs.

The estimates of travel time indicate that the users are more sensitive to travel time when they have to go through the center, in comparison with the other alternatives. This is also reflected in the length coefficient of the second model, where length is penalized more in the utility of the through the $C C$ alternative, compared with the generic coefficient for the other three alternatives. The piecewise specification of the travel time coefficients for the around alternatives reveals that users are less sensitive when the travel times are short (shorter than 10 minutes) and more sensitive when

\footnotetext{
${ }^{4}$ The models are estimated using Biogeme, an open source software for discrete choice models (Bierlaire, 2003).
} 
Table 3: Descriptive statistics on attributes

\begin{tabular}{c|ccccc}
\hline & mean & median & min & max & std. dev \\
\hline TT_CC (min) & 10.18 & 8.38 & 3.88 & 38.03 & 6.41 \\
TT_CL (min) & 9.98 & 8.18 & 2.86 & 38.93 & 6.32 \\
TT_CO (min) & 10.21 & 8.37 & 3.81 & 36.47 & 6.23 \\
TT_AV (min) & 11.80 & 13.12 & 2.66 & 38.58 & 11.81 \\
L_CC (km) & 7.65 & 5.21 & 1.88 & 42.91 & 7.39 \\
L_CL (km) & 7.84 & 5.47 & 1.57 & 43.82 & 7.30 \\
L_CO (km) & 7.95 & 5.48 & 2.33 & 42.62 & 7.23 \\
L_AV (km) & 9.18 & 9.04 & 1.54 & 42.29 & 8.90 \\
IS_CC (\#) & 27 & 26 & 9 & 51 & 9 \\
IS_CL (\#) & 25 & 24 & 6 & 58 & 10 \\
IS_CO (\#) & 26 & 26 & 4 & 48 & 11 \\
IS_AV (\#) & 34 & 37 & 10 & 75 & 15 \\
LT_CC (\#) & 2 & 2 & 0 & 8 & 2 \\
LT_CL (\#) & 2 & 2 & 0 & 5 & 1 \\
LT_CO (\#) & 2 & 2 & 0 & 4 & 1 \\
LT_AV (\#) & 3 & 3 & 0 & 9 & 2 \\
\hline
\end{tabular}

Table 4: Frequency of the chosen alternatives

\begin{tabular}{cc}
\hline Choice & \# times chosen \\
\hline CC & 13 \\
CL & 53 \\
CO & 51 \\
AV & 22 \\
\hline Total & 139 \\
\hline
\end{tabular}




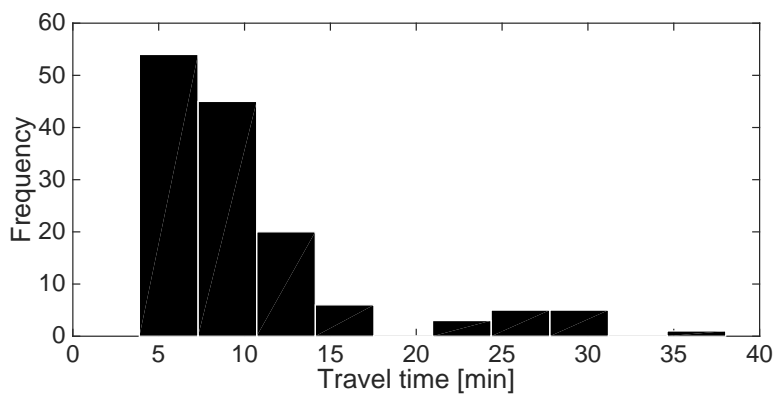

(a) $\mathrm{CC}$

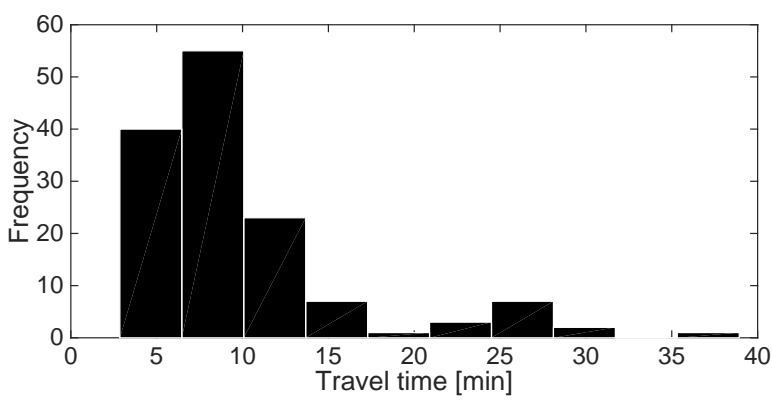

(b) CL

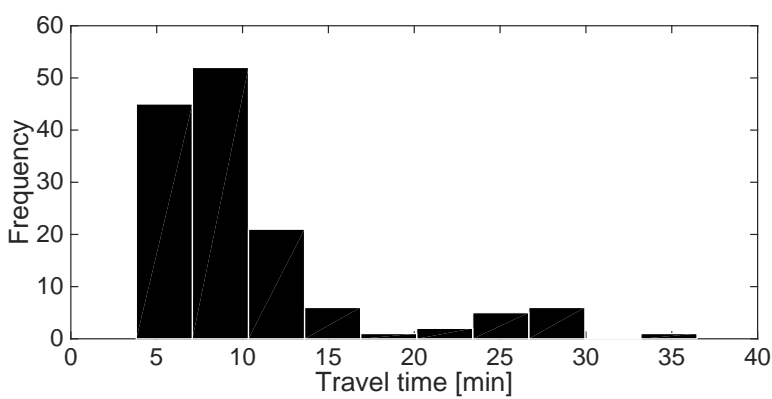

(c) $\mathrm{CO}$

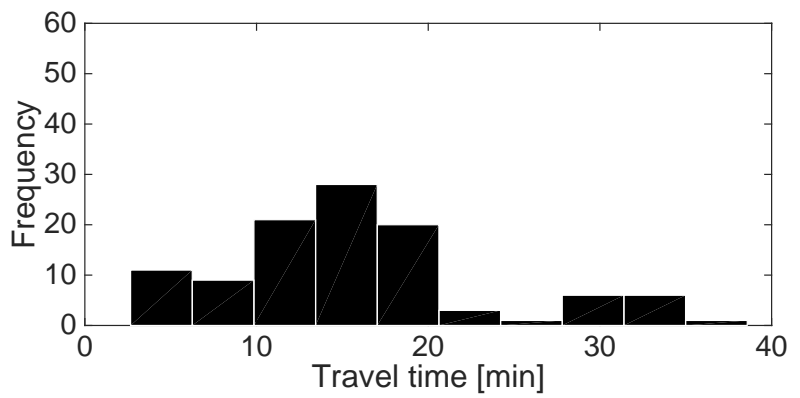

(d) AV

Figure 12: Distribution of travel time for the four MRIs. 
the travel time becomes longer. Looking at the two models, the parameter estimates for the number of left turns and the number of intersections are of equivalent magnitude.

Intuitively, the $\mathrm{CL}$ and $\mathrm{CO}$ alternatives may share unobserved attributes, and if this is the case they should be nested. Hence, a nested logit formulation, assuming a nest with the $\mathrm{CL}$ and $\mathrm{CO}$ alternatives, was also tested and rejected by the likelihood ratio test against the logit model.

Table 5: Estimation results

\begin{tabular}{|c|c|c|}
\hline Parameters & $\begin{array}{c}\text { Model } 1 \\
\text { Value (Rob. } t \text {-test } 0)\end{array}$ & $\begin{array}{c}\text { Model } 2 \\
\text { Value (Rob. } t \text {-test } 0)\end{array}$ \\
\hline $\mathrm{ASC}_{\mathrm{CL}, \mathrm{CO}}$ & $-2.110(-1.47)$ & $-0.975(-0.58)$ \\
\hline$A S C_{A V}$ & $1.870(0.89)$ & $0.307(0.18)$ \\
\hline$\beta \operatorname{TIME}_{\mathrm{CC}}$ & $-0.772(-2.82)$ & \\
\hline$\beta \operatorname{TIME}_{\mathrm{CL}, \mathrm{CO}}^{(0-10 \mathrm{~min})}$ & $-0.286(-1.74)$ & \\
\hline$\beta \operatorname{TIME}_{\mathrm{CL}, \mathrm{CO}}^{(>10 \mathrm{~min})}$ & $-0.616(-2.86)$ & \\
\hline$\beta T I M E_{A V}$ & $-0.583(-3.11)$ & \\
\hline$\beta$ LENGTH $_{C C}$ & & $-1.480(-2.99)$ \\
\hline$\beta$ LENGTH ${ }_{\mathrm{CL}, \mathrm{CO}, A V}$ & & $-0.871(-5.03)$ \\
\hline$\beta$ LEFT & $-0.288(2.22)$ & $-0.270(-1.89)$ \\
\hline$\beta \mathrm{IS}$ & $-0.047(-2.16)$ & $-0.063(-3.42)$ \\
\hline Number of observations & 139 & 139 \\
\hline Number of parameters & 8 & 6 \\
\hline $\bar{\rho}^{2}$ & 0.375 & 0.416 \\
\hline $\mathcal{L}(0)$ & -183.201 & -183.201 \\
\hline $\mathcal{L}(\widehat{\beta})$ & -106.563 & -101.064 \\
\hline
\end{tabular}




\subsection{Forecasting results \& validation}

In order to validate the model and test its performance with respect to the predicted choice probabilities, we adopt a cross-validation approach. The procedure is outlined below:

1. Randomly select $80 \%$ of the data for estimation.

2. Use the estimated model to predict the remaining $20 \%$.

3. Repeat 100 times.

The results are summarized in the box plot in Fig. 13, where we present the resulting sample shares for each alternative. In the same plot, we overlay the sample shares from the estimation on the full dataset (average probability), as well as their 5\% and $95 \%$ percentiles. The result is quite satisfying as it appears that the level of precision for the forecast, using the point estimates of the parameters, is consistent with the confidence interval of the full model considering the distribution of the estimators.

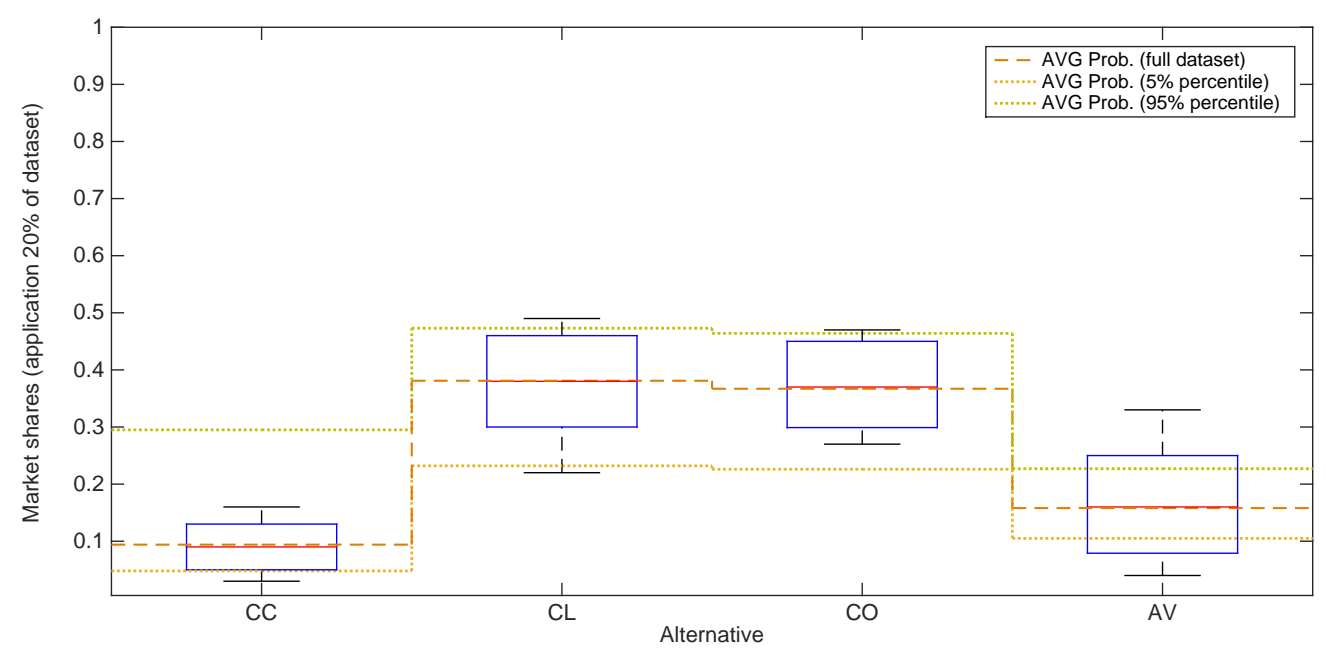

Figure 13: Box plot of the market shares from the application in $20 \%$ of the data against the confidence intervals from the estimation on the full dataset. 


\subsection{Model Application}

\subsubsection{Traffic assignment}

Following Section 3.5, and the specification of the model in Appendix B, we apply the MRI model to traffic assignment. For this example we take into account one OD pair of a person traveling from the south to the north of the city of Borlänge. The MRI choice probability $\mathrm{P}\left(i \mid \mathrm{C}_{n}\right)$ follows the specification given in Section 5.2. For the OD pair of interest we obtain: $\mathrm{P}(\mathrm{AV} \mid \mathcal{C})=0.002, \mathrm{P}(\mathrm{CC} \mid \mathcal{C})=0.084, \mathrm{P}(\mathrm{CL} \mid \mathcal{C})=0.247, \mathrm{P}(\mathrm{CO} \mid \mathcal{C})=$ 0.667 .

For the specification of $P(p \mid i)$ we have chosen $\alpha=25$ and $\beta=-2.5$. Figure 14 depicts for each link a of the network, the probability -as defined in (2) and approximated by (6) - that a single traveler executing a single trip passes through that link. On this figure one can identify the links where congestion might occur. Figures 15 to 18 show the link choice probabilities -as defined in (3) and approximated by (5)- conditional on the choice of the indicated MRI. On these figures one can identify the most attractive links for each MRI.

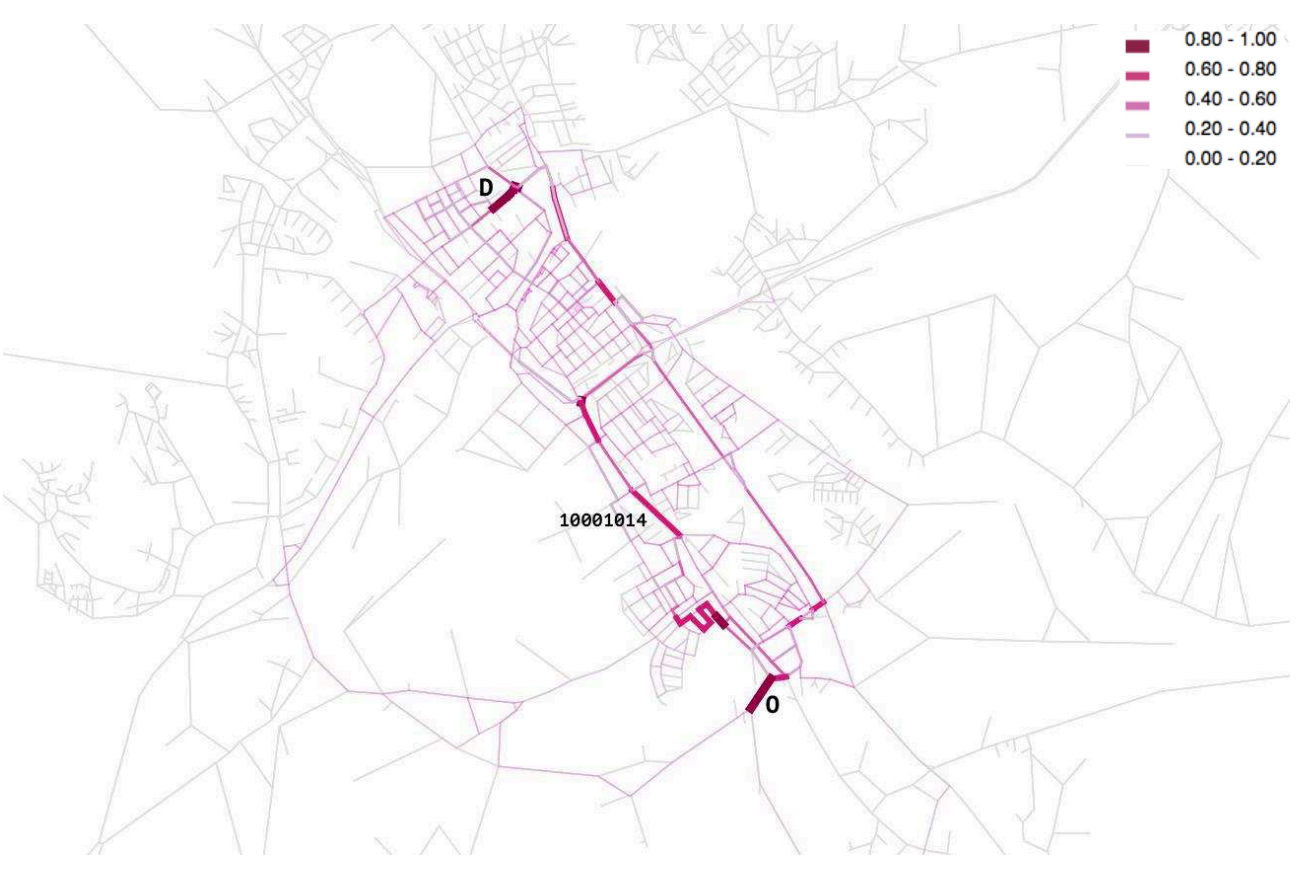

Figure 14: Link choice probabilities given the MRI choice set. 


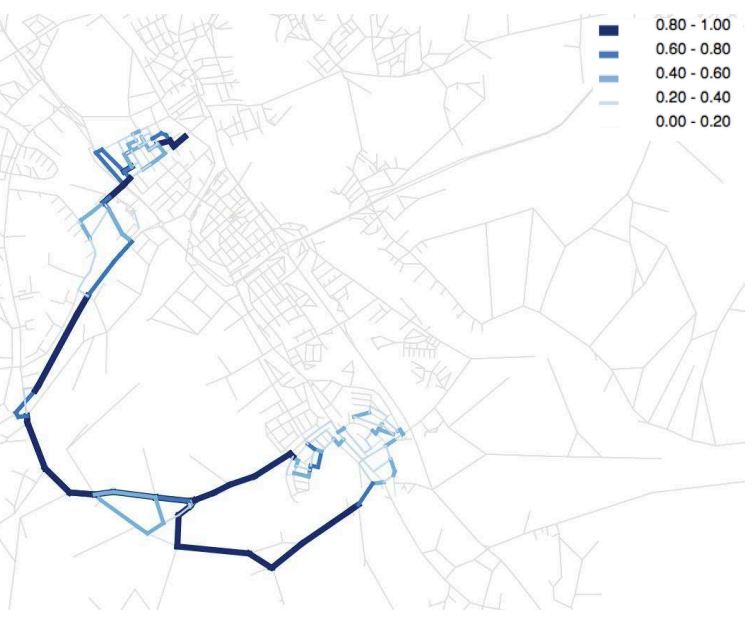

Figure 15: Link choice probabilities conditional on the choice of the avoid the CC alternative.

Figure 16: Link choice probabilities conditional on the choice of going through the $\mathrm{CC}$ alternative. 


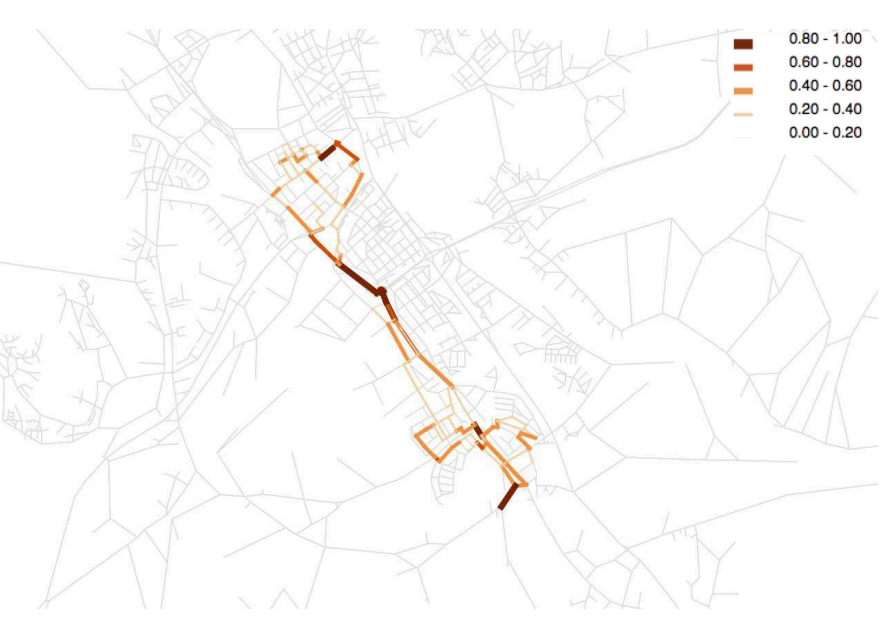

Figure 17: Link choice probabilities conditional on the choice of going around and clockwise the $\mathrm{CC}$ alternative.

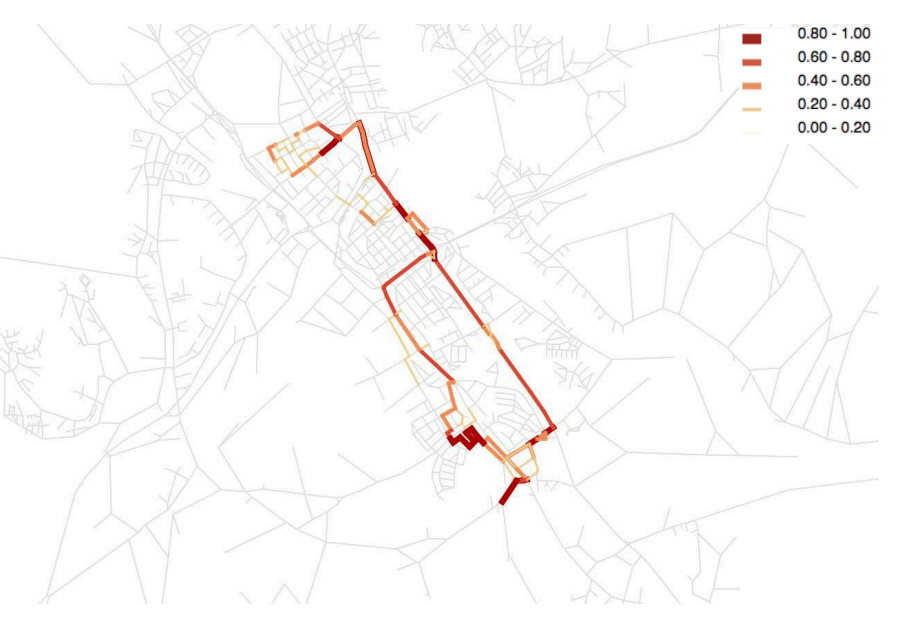

Figure 18: Link choice probabilities conditional on the choice of going around and counter-clockwise the $\mathrm{CC}$ alternative. 


\section{Conclusion}

In this paper, we present a new approach for route choice analysis that is designed to be flexible and simple. It explicitly separates the high level decisions, associated with mental representations, from the operational decisions resulting in explicit paths. The framework has to be adapted on a case by case basis. We have shown here, using a real case study with RP data, that the use of simple heuristics leads to a meaningful model that can be estimated and used in practice.

The proposed framework opens the door to more modeling opportunities. The MRI concept is sufficiently general to capture a great deal of behaviorally meaningful aspects of route choice, yet sufficiently specific to yield operational models. It also motivates new approaches to data collection. In particular, it would suggest to conduct specific surveys that would help the modeler in defining the MRIs and the perception of the performance of each alternative. New possibilities for the use of route choice models have been identified as well. Namely, the concept of MRI may be particularly relevant for travel information and guidance.

A great deal of future research is anticipated with this novel framework. Other case studies and model specifications should be investigated. We are currently considering the cross-nested logit (CNL) and the recursive logit $(\mathrm{RL})$ models. In the present work, we focus on a relatively small network and sample of observations. The next step is to test the methodology in a bigger network and data sample. 


\section{Acknowledgements}

This research is supported by the Swiss National Science Foundation Grant \#200021 - 146621 "Capturing latent concepts with non invasive sensing systems". The authors would like to thank Matthieu de Lapparent for his assistance and fruitful discussions. 


\section{References}

Arentze, T. a. and Timmermans, H. J. P. (2005). Representing mental maps and cognitive learning in micro-simulation models of activitytravel choice dynamics, Transportation 32(4): 321-340.

Axhausen, K. W., Schönfelder, S., Wolf, J. and Oliveira, M. (2003). 80 weeks of GPS-traces: Approaches to enriching the trip information, Transportation Research Record 1870: 46-54.

Azevedo, J., Santos Costa, M., Silvestre Madeira, J. and Vieira Martins, E. (1993). An algorithm for the ranking of shortest paths, European Journal of Operational Research 69(1): 97-106.

Bekhor, S., Ben-akiva, M. E. and Ramming, M. S. (2002). Adaptation of Logit Kernel to Route Choice Situation, Transportation Research Record: Journal of the Transportation Research Board pp. 78-85.

Bekhor, S., Ben-Akiva, M. E. and Ramming, S. M. (2006). Evaluation of choice set generation algorithms for route choice models, Annals of Operations Research 144(1): 235-247.

Ben-Akiva, M., Bergman, M. J., Daly, A. and Ramaswamy, V. (1984). Modeling interurban route choice behavior, Proceedings of the 9th International Symposium on Transportation and Traffic Theory, Utrecht, The Netherlands, pp. 299-330.

Ben-Akiva, M. and Bierlaire, M. (1999). Discrete choice methods and their applications to short-term travel decisions, in R. Hall (ed.), Handbook of Transportation Science, Operations Research and Management Science, Kluwer, pp. 5-34. ISBN:0-7923-8587-X.

Ben-Akiva, M. and Bierlaire, M. (2003). Discrete choice models with applications to departure time and route choice, in R. Hall (ed.), Handbook of Transportation Science, 2nd edition, Operations Research and Management Science, Kluwer, pp. 7-38. ISBN:1-4020-7246-5.

Bierlaire, M. (2003). BIOGEME: A free package for the estimation of discrete choice models, 3rd Swiss Transportation Research Conference, Ascona, Switzerland. 
Bierlaire, M. and Frejinger, E. (2008). Route choice modeling with networkfree data, Transportation Research Part C: Emerging Technologies 16(2): 187-198.

Boarnet, M., Kim, E. and Parkany, E. (1998). Modeling Route Choice Behavior: How Relevant Is the Composition of Choice Set?, Transportation Research Record: Journal of the Transportation Research Board 1634: 93-99.

Bovy, P. and Fiorenzo Catalano, S. (2007). Stochastic route choice set generation: behavioral and probabilistic foundations, Transportmetrica 3(3): 173-189.

Bovy, P. H. L. and Stern, E. (1990). Route Choice: Wayfinding in Transport Networks, Studies in Industrial Organization, Kluwer Academic Publishers.

Cascetta, E., Nuzzolo, A., Russo, F. and Vitetta, A. (1996). A modified logit route choice model overcoming path overlapping problems: specification and some calibration results for interurban networks, Proceedings of the Thirteenth International Symposium on Transportation and Traffic Theory, Lyon, France, pp. 697-711.

Chase, W. G. (1983). D. A. Rogers \& J. A. Sloboda (Eds.), The acquisition of symbolic skills, New York: Plenum, chapter Spatial representations of taxi drivers, pp. 391-405.

Chen, J. and Bierlaire, M. (2013). Probabilistic multimodal map-matching with rich smartphone data, Journal of Intelligent Transportation Systems .

Couclelis, H., Golledge, R. G., Gale, N. and Tobler, W. (1987). Exploring the anchor point hypothesis of spatial cognition, Journal of Environmental Psychology 7: 99-122.

Daganzo, C. F. and Sheffi, Y. (1977). On stochastic models of traffic assignment, Transportation Science 11: 253-274.

de la Barra, T., Perez, B. and Anez, J. (1993). Multidimensional path search and assignment., Proceedings of the 21st PTRC Summer Annual Meeting, Manchester, England. 
Dial, R. B. (1971). A probabilistic multipath traffic assignment model which obviates path enumeration, Transportation Research 5(2): 83 $-111$.

Flötteröd, G. and Bierlaire, M. (2013). Metropolis-Hastings sampling of paths, Transportation Research Part B: Methodological 48: 53-66.

Fosgerau, M., Frejinger, E. and Karlstrom, A. (2013). A link based network route choice model with unrestricted choice set, Transportation Research Part B: Methodological 56(0): $70-80$.

Fosgerau, M., McFadden, D. and Bierlaire, M. (2013). Choice probability generating functions, Journal of Choice Modelling 8: 1-18.

Frank, L. D., Sallis, J. F., Saelens, B. E., Leary, L., Cain, K., Conway, T. L. and Hess, P. M. (2009). The development of a walkability index: application to the Neighborhood Quality of Life Study, British Journal of Sports Medicine 44: 924-933.

Frejinger, E. (2008). Route choice analysis: data, models, algorithms and applications, PhD thesis, Ecole Polytechnique Fédérale de Lausanne, Switzerland.

Frejinger, E. and Bierlaire, M. (2007). Capturing correlation with subnetworks in route choice models, Transportation Research Part B: Methodological 41(3): 363-378.

Frejinger, E., Bierlaire, M. and Ben-Akiva, M. (2009). Sampling of alternatives for route choice modeling, Transportation Research Part B: Methodological 43(10): 984-994.

Friedrich, M., Hofsaess, I. and Wekeck, S. (2001). Timetable-based transit assignment using branch and bound techniques, Transportation Research Record: Journal of the Transportation Research Board pp. 100-107.

Golledge, R. G. (1999). Wayfinding Behavior: Cognitive Mapping and Other Spatial Processes, Johns Hopkins University Press, Baltimore, $\mathrm{MD}$. 
Golledge, R. G. and Gärling, T. (2003). Cognitive Maps and Urban Travel, University of California Transportation Center .

Guevara, C. A. and Ben-Akiva, M. E. (2013). Sampling of alternatives in multivariate extreme value (mev) models, Transportation Research Part B: Methodological 48(0): $31-52$.

Hannes, E., Janssens, D. and Wets, G. (2008). Destination Choice in Daily Activity Travel: Mental Map's Repertoire, Transportation Research Record: Journal of the Transportation Research Board 2054: 2027.

Hoogendoorn-Lanser, S. (2005). Modelling Travel Behavior in Multimodal Networks, PhD thesis, Technology University of Delft.

Lai, X. and Bierlaire, M. (2015). Specification of the cross-nested logit model with sampling of alternatives for route choice models, Transportation Research Part B: Methodological 80: $220-234$.

Lynch, K. (1960). The Image of the City, M.A.: MIT Press, Cambridge.

Mai, T., Fosgerau, M. and Frejinger, E. (2015). A nested recursive logit model for route choice analysis, Transportation Research Part B: Methodological 75: 100 - 112.

Prato, C. and Bekhor, S. (2007). Modeling Route Choice Behavior: How Relevant Is the Composition of Choice Set?, Transportation Research Record: Journal of the Transportation Research Board 2003: 6473.

Prato, C. G. and Bekhor, S. (2006). Applying branch and bound technique to route choice set generation, Transportation Research Record pp. 19-28.

Ramos, G. D. M. (2015). Dynamic Route Choice Modelling of the Effects of Travel Information using RP Data, $\mathrm{PhD}$ thesis, Delft University of Technology.

Suttles, G. (1972). The Social Construction of Communities, The University of Chicago Press, Chicago. 
Taylor, H. A. and Tversky, B. (1992). Spatial mental models derived from survey and route descriptions, Journal of Memory and Language 31(2): $261-292$.

Tolman, E. C. (1948). Cognitive maps in rats and men, Psychological Review 55: 189-208.

van der Zijpp, N. J. and Fiorenzo Catalano, S. (2005). Path enumeration by finding the constrained K-shortest paths, Transportation Research Part B: Methodological 39(6): 545-563.

Vovsha, P. and Bekhor, S. (1998). Link-Nested Logit Model of Route Choice: Overcoming Route Overlapping Problem, Transportation Research Record: Journal of the Transportation Research Board 1645: 133-142.

Yang, J. and Juang, G. (2014). Development of an enhanced route choice model based on cumulative prospect theory, Transportation Research Part C: Emerging Technologies 47, Part 2(0): 168-178. 


\section{A Description of a work trip in Athens' road network}

This appendix provides a qualitative example in support of the MRI assumption. The case study area is located in Athens, Greece. Athens is a big city and its transportation network is very dense and complicated. The characteristics of the driver are:

- Male;

- 54 years old;

- Familiar with the route as he has been doing this trip for more than 20 years;

- Experienced driver with very good knowledge of the network (living in Athens all his life and driving from the age of 18);

- He either uses a motorcycle or a car for his transportation.

Figure A1 depicts all the relevant components that we discuss hereafter. We asked the driver to describe his regular route from home to work. He said that there are two alternatives: either to go through the city center (red-shaded area) or to take the peripheral road (green polyline).

After asking the driver to give more details regarding the two itineraries, he described different options associated to each of these two alternatives. Given that he chooses to go through the peripheral he has several ways to reach it: (i) neighbourhood $Y$ and then neighbourhood $V$, or (ii) neighbourhood I. Continuing describing his route, he stated that after exiting the peripheral and a while before reaching the destination he has another two options: (i) either through a main arterial (extension of the green line), or (ii) through cutting though neighbourhood $\mathrm{P}$ (blue line). The name of the peripheral road (Katechaki) came up several times during the description as it is associated with all of these itineraries.

When he chooses to go through the city centre, he regularly follows one specific itinerary along main arterials. He only pointed one minor deviation to a minor street in order to avoid a traffic light in cases of congestion. After being explicitly asked, the driver mentioned that he adjusts his itinerary in the level of minor streets, mainly due to bottlenecks that he may encounter. In this case though, he did not refer to the exact options. 
Being asked what defines his choice, he said that it depends on his mood and on the current traffic conditions. He prefers to go through the city centre as it is more pleasant for him than the peripheral, that he called monotonous. The city centre alternative is also the shorter option with respect to kilometres, but it is usually more congested in the morning. For this reason he usually takes the peripheral road on his way to work. On the other hand, on his way back home in the evening he always chooses to go through the centre as it is not congested during this time of day. It worths noticing that apart from abstraction in the representation of the possible alternatives, there is abstraction in the association of attributes to them. It is highlighted by the use of adjectives, like fast, pleasant etc.

While talking, the respondent made use of major streets' names and neighbourhoods as described above, but he also referred to schools in one of the neighbourhoods, park in another, squares, an ancient stadium which is a landmark in Athens, a cemetery (the first and biggest of the city), several churches that are located along these routes, a cinema, the tower of Athens, and also home location of friends and relatives that signify reference points (anchor points and landmarks) along the way. When asked if locations such as the stadium play any role in his choice of route the answer was: "No, I just meet them on my way". These elements facilitate the description of his route. They are used to improve communication and understanding, and they characterize the alternative routes. It should be underlined that there is a distinction between these reference points and landmarks along the way and the representations that consist alternatives. The former though can be used to construct explanatory variables. For instance, areas with increased number of landmarks and points of interest might be more attractive, or more familiar, to travelers. They may also serve as representative points for the definition of the MRIs that we discuss in this paper. 


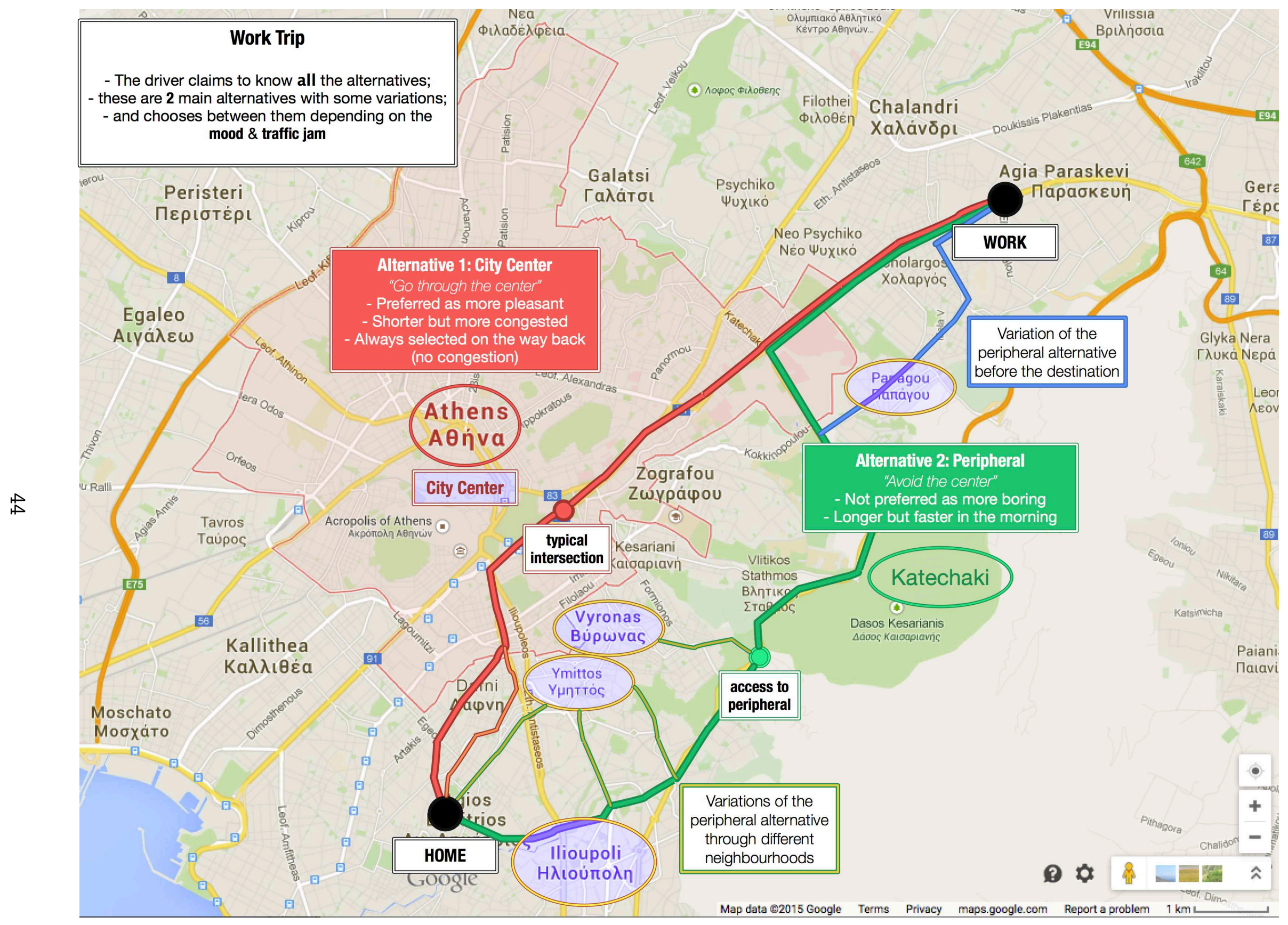

Figure A1: Sketch of the described route alternatives. 


\section{B Specification of the assignment model}

This appendix presents a simple example for the specification of the assignment model (equation 2).

Let $s_{v}^{i}$ be a real number representing the consistency of node $v$ with MRI i. The determination of $s_{v}^{i}$ reflects the definition of the MRIs on the basis of the geographical span and the representative points. In particular, if a node is contained in the MRI's geographical span it has a consistency equal to 1 , and 0 otherwise. The nodes corresponding to the representative points of a MRI may receive a consistency value higher than 1 .

Each path $p$ consists in a sequence of nodes. We can then compute the score $s_{p}^{i}=\sum_{v \in p} s_{v}^{i}$ of each path for every MRI $i \in \mathcal{C}_{n}$, where $\sum_{v \in p}$. represents the sum over all nodes $v$ contained in path $p . s_{p}^{i}$ denotes the consistency of a path $p$ with a MRI $i$.

The path choice probability from the universal path choice set given a MRI $i$ is then, apart from normalization, specified as

$$
P(p \mid i) \sim \exp \left(\alpha \frac{s_{p}^{i}}{\sum_{j \in \mathcal{C}_{n}} s_{p}^{j}}+\beta t_{p}\right)
$$

where $\sum_{j \in \mathcal{C}_{n}} \cdot$ spans over all MRIs in $\mathcal{C}_{n}, t_{p}$ is the travel time on path $p$, and $\alpha>0, \beta<0$ are real-valued coefficients. The consistency sum favors paths that have relatively high node overlap with MRI $i$. It represents, at the path level, the traveler's operational decisions leading at the linkby-link level to an implementation of MRI $i$. The second factor favors paths that are faster. Some cost-dependency of this type is needed because otherwise it becomes more important to stay in the MRI than to reach the destination.

Now, the link choice probabilities defined in (2) need to be computed. The number of paths with nonzero probability of being selected given that MRI $i$ was chosen may be too high to be enumerated and used in the traffic assignment context. We propose to use the Metropolis-Hastings Algorithm of Flötteröd and Bierlaire (2013) to draw, for each MRI i, a large number of $Q_{i}$ paths from the un-normalized distribution (4). Letting $p_{i}^{q}$ be the $q$ th path drawn for MRI $i, P(a \mid i)$ is then approximated by

$$
\widehat{\mathrm{P}}(\mathrm{a} \mid \mathrm{i})=\frac{1}{\mathrm{Q}_{\mathrm{i}}} \sum_{\mathrm{q}=1}^{\mathrm{Q}_{i}} \mathbf{1}\left(a \in p_{i}^{\mathrm{q}}\right) \text {. }
$$


This is, for a given MRI $i$, the ratio of the number of times link $a$ is contained in a sampled path divided by the total number of sampled paths. The MRI-unconditional link choice probabilities (2) are then approximated through

$$
\widehat{\mathrm{P}}\left(\mathrm{a} \mid \mathrm{C}_{\mathrm{n}}, x_{i n}, z_{n}\right)=\sum_{i \in \mathcal{C}_{n}} \hat{\mathrm{P}}(\mathrm{a} \mid \mathfrak{i}) \cdot \mathrm{P}\left(\mathfrak{i} \mid \mathrm{C}_{n}, x_{i n}, z_{\mathfrak{n}}\right) .
$$




\section{Additional Figures}




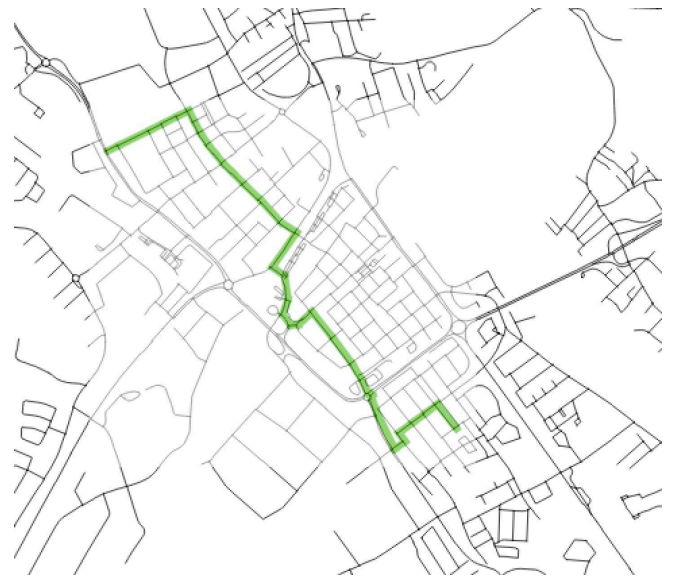

(a) Through CC.

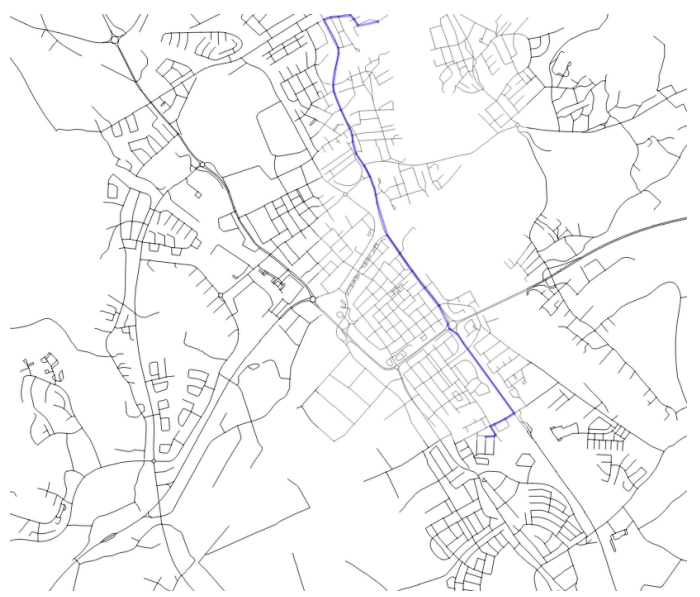

(c) Clockwise CC.

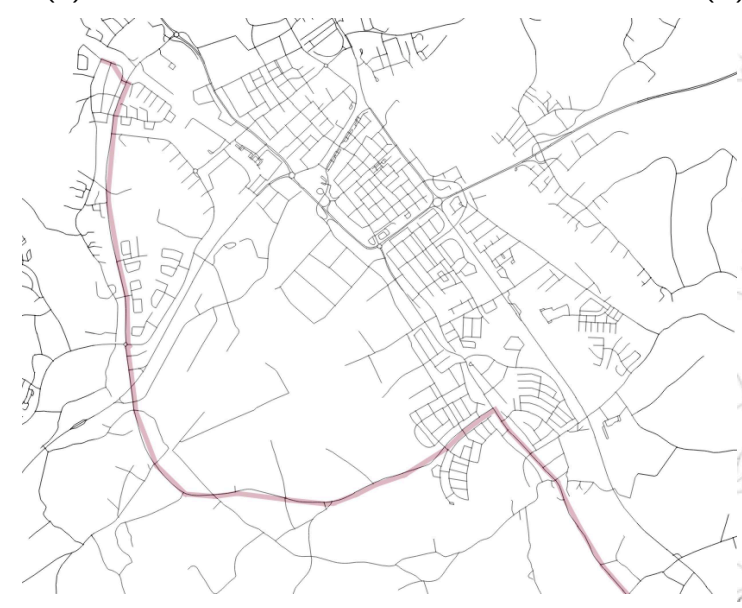

(e) Avoid CC.

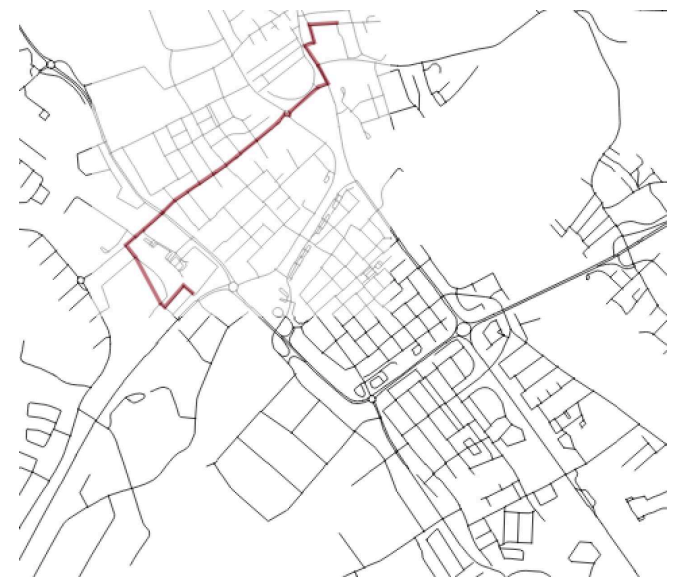

(b) Avoid CC.

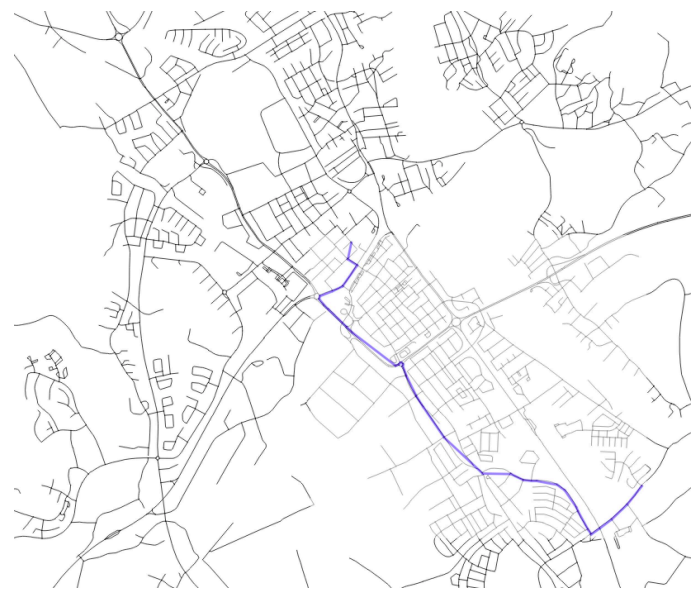

(d) Counter-clockwise CC.

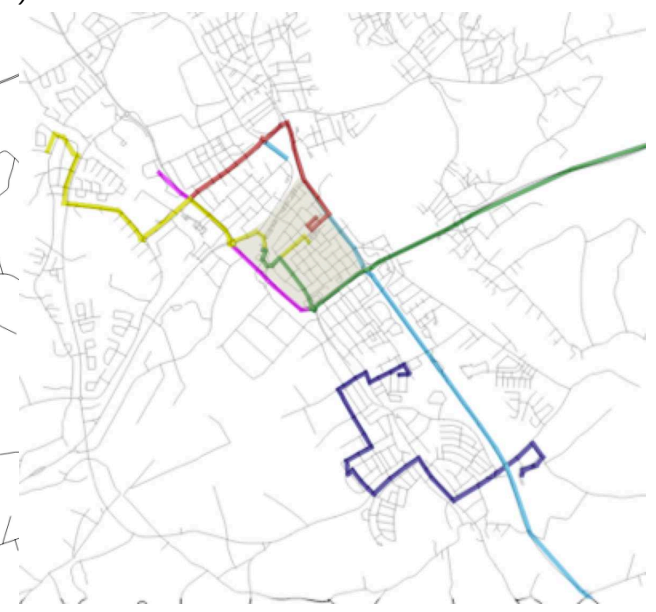

(f) Various.

Figure C1: Characteristic examples of observed trajectories. 